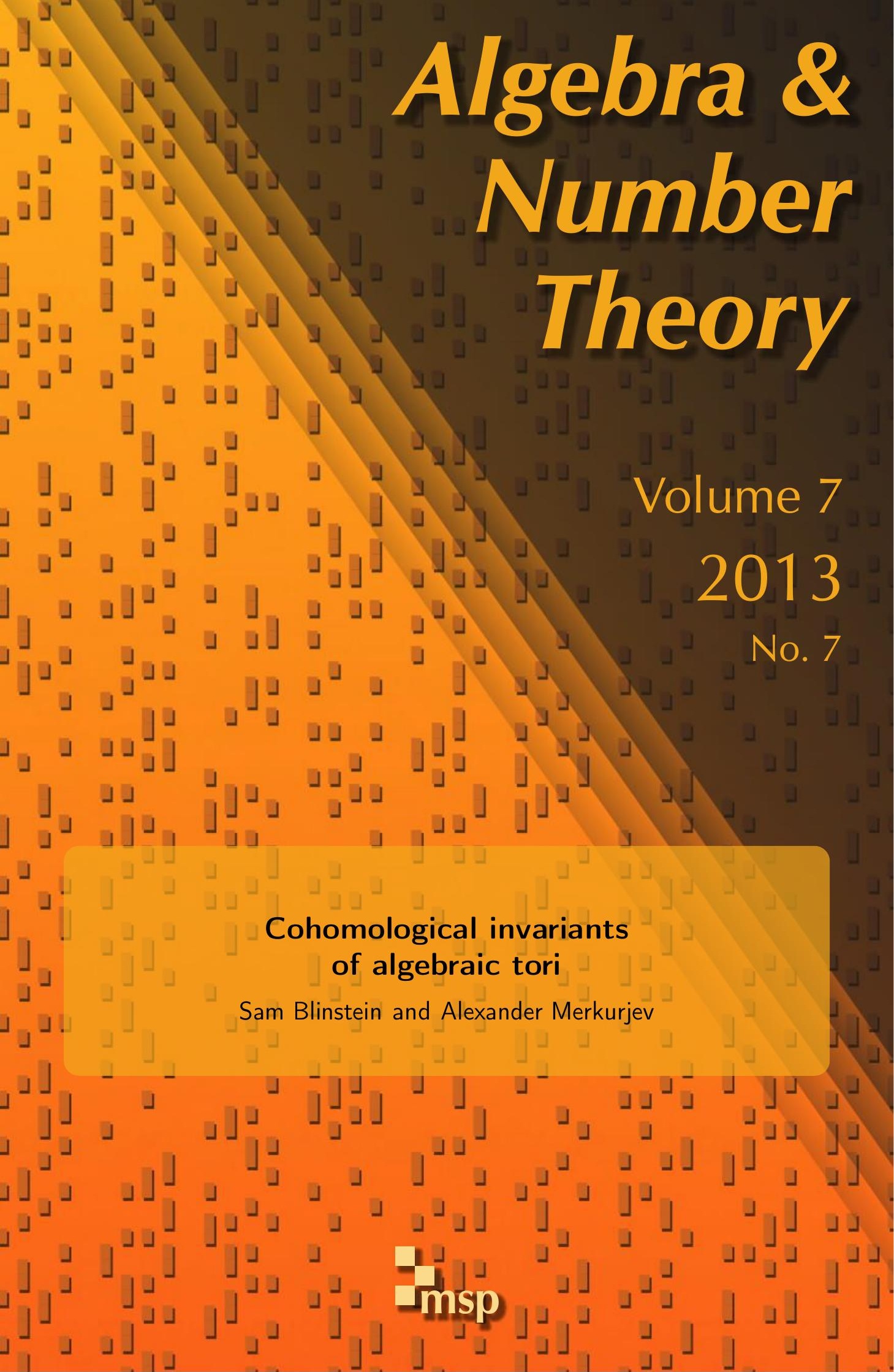




\title{
Cohomological invariants of algebraic tori
}

\author{
Sam Blinstein and Alexander Merkurjev
}

Let $G$ be an algebraic group over a field $F$. As defined by Serre, a cohomological invariant of $G$ of degree $n$ with values in $\mathbb{Q} / \mathbb{Z}(j)$ is a functorial-in- $K$ collection of maps of sets $\operatorname{Tors}_{G}(K) \rightarrow H^{n}(K, \mathbb{Q} / \mathbb{Z}(j))$ for all field extensions $K / F$, where $\operatorname{Tors}_{G}(K)$ is the set of isomorphism classes of $G$-torsors over Spec $K$. We study the group of degree 3 invariants of an algebraic torus with values in $\mathbb{Q} / \mathbb{Z}(2)$. In particular, we compute the group $H_{\mathrm{nr}}^{3}(F(S), \mathbb{Q} / \mathbb{Z}(2))$ of unramified cohomology of an algebraic torus $S$.

\section{Introduction}

Let $G$ be a linear algebraic group over a field $F$ (of arbitrary characteristic). The notion of an invariant of $G$ was defined in [Garibaldi et al. 2003] as follows. Consider the category Fields $F$ of field extensions of $F$ and the functor

$$
\operatorname{Tors}_{G}: \text { Fields }_{F} \rightarrow \text { Sets }
$$

taking a field $K$ to the set $\operatorname{Tors}_{G}(K)$ of isomorphism classes of (right) $G$-torsors over Spec $K$. Let

$$
H: \text { Fields }_{F} \rightarrow \text { Abelian Groups }
$$

be another functor. An $H$-invariant of $G$ is then a morphism of functors

$$
i: \operatorname{Tors}_{G} \rightarrow H,
$$

viewing $H$ with values in Sets, that is, a functorial in $K$ collection of maps of sets $\operatorname{Tors}_{G}(K) \rightarrow H(K)$ for all field extensions $K / F$. We denote the group of $H$-invariants of $G$ by $\operatorname{Inv}(G, H)$.

An invariant $i \in \operatorname{Inv}(G, H)$ is called normalized if $i(I)=0$ for the trivial $G$-torsor $I$. The normalized invariants form a $\operatorname{subgroup} \operatorname{Inv}(G, H)_{\text {norm }} \operatorname{of} \operatorname{Inv}(G, H)$ and there is a natural isomorphism

The work of the second author has been supported by the NSF grant DMS \#1160206. MSC2010: primary 11E72; secondary 12G05.

Keywords: algebraic tori, cohomological invariants, Galois cohomology. 


$$
\operatorname{Inv}(G, H) \simeq H(F) \oplus \operatorname{Inv}(G, H)_{\text {norm }},
$$

so it is sufficient to study normalized invariants.

Typically, $H$ is a cohomological functor given by Galois cohomology groups with values in a fixed Galois module. Of particular interest to us is the functor $H$ which takes a field $K / F$ to the Galois cohomology group $H^{n}(K, \mathbb{Q} / \mathbb{Z}(j))$, where the coefficients $\mathbb{Q} / \mathbb{Z}(j)$ are defined as follows. For a prime integer $p$ different from the characteristic of $F$, the $p$-component $\mathbb{Q}_{p} / \mathbb{Z}_{p}(j)$ of $\mathbb{Q} / \mathbb{Z}(j)$ is the colimit over $n$ of the étale sheaves $\mu_{p^{n}}^{\otimes j}$, where $\mu_{m}$ is the sheaf of $m$-th roots of unity. In the case $p=\operatorname{char}(F)>0, \mathbb{Q}_{p} / \mathbb{Z}_{p}(j)$ is defined via logarithmic de Rham-Witt differentials; see Section $3 b$.

We write $\operatorname{Inv}^{n}(G, \mathbb{Q} / \mathbb{Z}(j))$ for the group of cohomological invariants of $G$ of degree $n$ with values in $\mathbb{Q} / \mathbb{Z}(j)$.

The second cohomology group $H^{2}(K, \mathbb{Q} / \mathbb{Z}(1))$ is canonically isomorphic to the Brauer group $\operatorname{Br}(K)$ of the field $K$. In Section 2c we prove (Theorem 2.4) that if $G$ is a connected group (reductive if $F$ is not perfect), then

$$
\operatorname{Inv}(G, \mathrm{Br})_{\text {norm }} \simeq \operatorname{Pic}(G) .
$$

The group $\operatorname{Inv}^{3}(G, \mathbb{Q} / \mathbb{Z}(2))_{\text {norm }}$ for a semisimple simply connected group $G$ has been studied by Rost; see [Garibaldi et al. 2003].

An essential object in the study of cohomological invariants is the notion of a classifying torsor: a $G$-torsor $E \rightarrow X$ for a smooth variety $X$ over $F$ such that every $G$-torsor over an infinite field $K / F$ is isomorphic to the pull-back of $E \rightarrow X$ along a $K$-point of $X$. If $V$ is a generically free linear representation of $G$ with a nonempty open subset $U \subset V$ such that there is a $G$-torsor $\pi: U \rightarrow X$, then $\pi$ is classifying. Such representations exist (see Section $2 b$ ).

The generic fiber of $\pi$ is the generic torsor over $\operatorname{Spec} F(X)$ attached to $\pi$. Evaluation at the generic torsor yields a homomorphism

$$
\operatorname{Inv}^{n}(G, \mathbb{Q} / \mathbb{Z}(j)) \rightarrow H^{n}(F(X), \mathbb{Q} / \mathbb{Z}(j)),
$$

and in Section 3 we show that the image of this map is contained in the subgroup $H_{\mathrm{Zar}}^{0}\left(X, \mathscr{H}^{n}(\mathbb{Q} / \mathbb{Z}(j))\right)$ of $H^{n}(F(X), \mathbb{Q} / \mathbb{Z}(j))$, where $\mathscr{H}^{n}(\mathbb{Q} / \mathbb{Z}(j))$ is the Zariski sheaf associated to the presheaf $W \mapsto H^{n}(W, \mathbb{Q} / \mathbb{Z}(j))$ of the étale cohomology groups. In fact, the image is contained in the subgroup $H_{\mathrm{Zar}}^{0}\left(X, \mathscr{H}^{n}(\mathbb{Q} / \mathbb{Z}(j))\right)_{\text {bal }}$ of balanced elements, that is, elements that have the same images under the pull-back homomorphisms with respect to the two projections $(U \times U) / G \rightarrow X$. Moreover, the balanced elements precisely describe the image and we prove (Theorem 3.4):

Theorem A. Let $G$ be a smooth linear algebraic group over a field $F$. We assume that $G$ is connected if $F$ is a finite field. Let $E \rightarrow X$ be a classifying $G$-torsor with 
$E$ a $G$-rational variety such that $E(F) \neq \varnothing$. Then (1-1) yields an isomorphism $\operatorname{Inv}^{n}(G, \mathbb{Q} / \mathbb{Z}(j)) \simeq H_{\mathrm{Zar}}^{0}\left(X, \mathscr{H}^{n}(\mathbb{Q} / \mathbb{Z}(j))\right)_{\mathrm{bal}}$.

At this point it is convenient to make use of a construction due to Totaro [1999]: because the Chow groups are homotopy invariant, the groups $\mathrm{CH}^{n}(X)$ do not depend on the choice of the representation $V$ and the open set $U \subset V$ provided the codimension of $V \backslash U$ in $V$ is large enough. This leads to the notation $\mathrm{CH}^{n}(B G)$, the Chow groups of the so-called classifying space $B G$, although $B G$ itself is not defined in this paper.

Unfortunately, the étale cohomology groups with values in $\mathbb{Q}_{p} / \mathbb{Z}_{p}(j)$, where $p=\operatorname{char}(F)>0$, are not homotopy invariant. In particular, we cannot use the theory of cycle modules of Rost [1996].

The main result of this paper is the exact sequence in Theorem 4.3 describing degree 3 cohomological invariants of an algebraic torus $T$. Writing $\widehat{T}_{\text {sep }}$ for the character lattice of $T$ over a separable closure of $F$ and $T^{\circ}$ for the dual torus, we prove our main result:

Theorem B. Let $T$ be an algebraic torus over a field $F$. Then there is an exact sequence

$$
\begin{aligned}
0 \rightarrow \mathrm{CH}^{2}(B T)_{\text {tors }} \rightarrow H^{1}\left(F, T^{0}\right) \stackrel{\alpha}{\rightarrow} \operatorname{Inv}^{3}(T, \mathbb{Q} / \mathbb{Z}(2))_{\text {norm }} \\
\quad \rightarrow H^{0}\left(F, S^{2}\left(\widehat{T}_{\mathrm{sep}}\right)\right) / \operatorname{Dec} \rightarrow H^{2}\left(F, T^{0}\right) .
\end{aligned}
$$

The homomorphism $\alpha$ is given by $\alpha(a)(b)=a_{K} \cup b$ for every $a \in H^{1}\left(F, T^{0}\right)$ and $b \in H^{1}(K, T)$ and every field extension $K / F$, where the cup-product is defined in (4-5), and Dec is the subgroup of decomposable elements in the symmetric square $S^{2}\left(\widehat{T}_{\text {sep }}\right)$ defined in Section A-II.

In the proof of the theorem we compute the group of balanced elements in the motivic cohomology group $H^{4}(B T, \mathbb{Z}(2))$ and relate it, using an exact sequence of B. Kahn and Theorem A, with the group of invariants $\operatorname{Inv}^{3}(T, \mathbb{Q} / \mathbb{Z}(2))_{\text {norm. }}$.

We also prove that the torsion group $\mathrm{CH}^{2}(B T)_{\text {tors }}$ is finite of exponent 2 (Theorem 4.7) and the last homomorphism in the sequence is also of exponent 2 (see the discussion before Theorem 4.13).

Moreover, if $p$ is an odd prime, the group $\operatorname{Inv}^{3}\left(T, \mathbb{Q}_{p} / \mathbb{Z}_{p}(2)\right)_{\text {norm }}$, which is the $p$-primary component of $\operatorname{Inv}^{3}(T, \mathbb{Q} / \mathbb{Z}(2))_{\text {norm }}$, splits canonically into the direct sum of linear invariants (those that induce group homomorphisms from Tors $T$ to $H^{3}$ ) and quadratic invariants, that is, the invariants $i$ such that the function $h(a, b):=i(a+b)-i(a)-i(b)$ is bilinear and $h(a, a)=2 i(a)$ for all $a$ and $b$. Furthermore, the groups of linear and quadratic invariants with values in $\mathbb{Q}_{p} / \mathbb{Z}_{p}(2)$ are canonically isomorphic to $H^{1}\left(F, T^{\circ}\right)\{p\}$ and $\left(H^{0}\left(F, S^{2}\left(\widehat{T}_{\text {sep }}\right)\right) / \operatorname{Dec}\right)\{p\}$, respectively. 
We also prove (Theorem 4.10) that the degree 3 invariants have control over the structure of all invariants. Precisely, the group $\operatorname{Inv}^{3}\left(T_{K}, \mathbb{Q} / \mathbb{Z}(2)\right)_{\text {norm }}$ is trivial for all $K / F$ if and only if $T$ is special, that is, $T$ has no nontrivial torsors over any field $K / F$, which in particular means $T$ has no nonconstant $H$-invariants for every functor $H$.

Our motivation for considering invariants of tori comes from their connection with unramified cohomology (defined in Section 5). Specifically, this work began as an investigation of a problem posed by Colliot-Thélène [1995, p. 39]: for $n$ prime to $\operatorname{char}(F)$ and $i \geq 0$, determine the unramified cohomology group $H_{\mathrm{nr}}^{i}\left(F(S), \mu_{n}^{\otimes(i-1)}\right)$, where $F(S)$ is the function field of a torus $S$ over $F$. The connection is provided by Theorem 5.7 where we show that the unramified cohomology of a torus $S$ is calculated by the invariants of an auxiliary torus:

Theorem C. Let $S$ be a torus over $F$ and let $1 \rightarrow T \rightarrow P \rightarrow S \rightarrow 1$ be a flasque resolution of $S$, that is, $T$ is flasque and $P$ is quasisplit. Then there is a natural isomorphism

$$
H_{\mathrm{nr}}^{n}(F(S), \mathbb{Q} / \mathbb{Z}(j)) \simeq \operatorname{Inv}^{n}(T, \mathbb{Q} / \mathbb{Z}(j)) .
$$

By Theorem B and Theorem C, we have an exact sequence

$$
\begin{aligned}
0 \rightarrow \mathrm{CH}^{2}(B T)_{\text {tors }} \rightarrow H^{1}\left(F, T^{0}\right) \stackrel{\alpha}{\rightarrow} \bar{H}_{\mathrm{nr}}^{3}( & F(S), \mathbb{Q} / \mathbb{Z}(2)) \\
& \rightarrow H^{0}\left(F, S^{2}\left(\widehat{T}_{\mathrm{sep}}\right)\right) / \operatorname{Dec} \rightarrow H^{2}\left(F, T^{0}\right)
\end{aligned}
$$

describing the reduced third cohomology group

$$
\left.\bar{H}_{\mathrm{nr}}^{3}(F(S), \mathbb{Q} / \mathbb{Z}(2))\right):=H_{\mathrm{nr}}^{3}(F(S), \mathbb{Q} / \mathbb{Z}(2)) / H^{3}(F, \mathbb{Q} / \mathbb{Z}(2)) .
$$

Moreover, for an odd prime $p$, we have a canonical direct sum decomposition of the $p$-primary components:

$$
\bar{H}_{\mathrm{nr}}^{3}\left(F(S), \mathbb{Q}_{p} / \mathbb{Z}_{p}(2)\right)=H^{1}\left(F, T^{0}\right)\{p\} \oplus\left(H^{0}\left(F, S^{2}\left(\widehat{T}_{\mathrm{sep}}\right)\right) / \operatorname{Dec}\right)\{p\} .
$$

Note that the torus $S$ determines $T$ up to multiplication by a quasisplit torus. If $X$ is a smooth compactification of $S$, one can take the torus $T$ with $\widehat{T}_{\text {sep }}=\operatorname{Pic}\left(X_{\text {sep }}\right)$; see [Colliot-Thélène and Sansuc 1977, §2].

In the present paper, $F$ denotes a field of arbitrary characteristic, $F_{\text {sep }}$ a separable closure of $F$, and $\Gamma$ the absolute Galois group $\operatorname{Gal}\left(F_{\text {sep }} / F\right)$ of $F$.

The word "scheme" over a field $F$ means a separated scheme over $F$ and, following [Fulton 1984], a "variety" over $F$ is an integral scheme of finite type over $F$. If $X$ is a scheme over $F$ and $L / F$ is a field extension then we write $X_{L}$ for $X \times{ }_{F}$ Spec $L$. When $L=F_{\text {sep }}$ we write simply $X_{\text {sep }}$.

A "linear algebraic group over $F$ " is an affine group scheme of finite type over $F$, not necessarily smooth. 


\section{Invariants of algebraic groups}

2a. Definitions and basic properties. Let $G$ be a linear algebraic group over a field $F$. Consider the functor

$$
\operatorname{Tors}_{G}: \text { Fields }_{F} \rightarrow \text { Sets }
$$

from the category of field extensions of $F$ to the category of sets taking a field $K$ to the set $\operatorname{Tors}_{G}(K)$ of isomorphism classes of (right) $G$-torsors over Spec $K$. Note that if $G$ is a smooth group, then there is a natural bijection

$$
\operatorname{Tors}_{G}(K) \simeq H^{1}(K, G):=H^{1}\left(\operatorname{Gal}\left(K_{\mathrm{sep}} / K\right), G\left(K_{\mathrm{sep}}\right)\right) .
$$

Let $H:$ Fields $_{F} \rightarrow$ Abelian Groups be a functor. We also view $H$ as a functor with values in Sets. Following [Garibaldi et al. 2003], we define an $H$-invariant of $G$ as a morphism of functors $\operatorname{Tors}_{G} \rightarrow H$ from the category Fields $s_{F}$ to Sets. All the $H$-invariants of $G$ form the abelian group of invariants $\operatorname{Inv}(G, H)$.

An invariant $i \in \operatorname{Inv}(G, H)$ is called constant if there is an element $h \in H(F)$ such that $i(I)=h_{K}$ for every $G$-torsor $I \rightarrow \operatorname{Spec} K$, where $h_{K}$ is the image of $h$ under natural map $H(F) \rightarrow H(K)$. The constant invariants form a subgroup $\operatorname{Inv}(G, H)_{\text {const }}$ of $\operatorname{Inv}(G, H)$ isomorphic to $H(F)$. An invariant $i \in \operatorname{Inv}(G, H)$ is called normalized if $i(I)=0$ for the trivial $G$-torsor $I$. The normalized invariants form a subgroup $\operatorname{Inv}(G, H)_{\text {norm }} \operatorname{of} \operatorname{Inv}(G, H)$ and we have the decomposition

$$
\operatorname{Inv}(G, H)=\operatorname{Inv}(G, H)_{\mathrm{const}} \oplus \operatorname{Inv}(G, H)_{\mathrm{norm}} \simeq H(F) \oplus \operatorname{Inv}(G, H)_{\mathrm{norm}},
$$

so it suffices to determine the normalized invariants.

2b. Classifying torsors. Let $G$ be a linear algebraic group over a field $F$. A $G$ torsor $E \rightarrow X$ over a smooth variety $X$ over $F$ is called classifying if for every field extension $K / F$, with $K$ infinite, and for every $G$-torsor $I \rightarrow \operatorname{Spec} K$, there is a point $x:$ Spec $K \rightarrow X$ such that the torsor $I$ is isomorphic to the fiber $E(x)$ of $E \rightarrow X$ over $x$, that is, $I \simeq E(x):=x^{*}(E)=\operatorname{Spec}(K) \times{ }_{X} E$. The generic fiber $E_{\text {gen }} \rightarrow \operatorname{Spec} F(X)$ of a classifying torsor is called a generic $G$-torsor; see [ibid., Part $1, \S 5.3]$.

If $V$ is a generically free linear representation of $G$ with a nonempty open subset $U \subset V$ such that there is a $G$-torsor $\pi: U \rightarrow X$, then $\pi$ is classifying; see [ibid., Part $1, \S 5.4]$. We will write $U / G$ for $X$ and call $\pi$ a standard classifying $G$-torsor. Standard classifying $G$-torsors exist: we can embed $G$ into $U:=\mathbf{G L}_{n, F}$ for some $n$ as a closed subgroup. Then $U$ is an open subset in the affine space $M_{n}(F)$ on which $G$ acts linearly and the canonical morphism $U \rightarrow X:=U / G$ is a $G$-torsor. Note that $U(F) \neq \varnothing$.

We say that a $G$-variety $Y$ is $G$-rational if there is an affine space $V$ with a linear $G$-action such that $Y$ and $V$ have $G$-isomorphic nonempty open $G$-invariant 
subvarieties. Note that if $U \rightarrow U / G$ is a standard classifying $G$-torsor, then $U$ is a $G$-rational variety.

Let $E \rightarrow X$ be a classifying $G$-torsor and let $H:$ Fields $_{F} \rightarrow$ Abelian Groups be a functor. Define the map

$$
\theta_{G}: \operatorname{Inv}(G, H) \rightarrow H(F(X)), \quad i \mapsto i\left(E_{\text {gen }}\right),
$$

by sending an invariant to its value at the generic torsor $E_{\text {gen }}$.

Consider the following property of the functor $H$ :

Property 2.1. The map $H(K) \rightarrow H(K((t)))$ is injective for any field extension $K / F$.

The following theorem, due to M. Rost, was proved in [Garibaldi et al. 2003, Part II, Theorem 3.3]. For completeness, we give a slightly modified proof in Section A-I.

Theorem 2.2. Let $G$ be a smooth linear algebraic group over $F$. If a functor $H:$ Fields $_{F} \rightarrow$ Abelian Groups has Property 2.1, then the map $\theta_{G}$ is injective, that is, every $H$-invariant of $G$ is determined by its value at the generic $G$-torsor.

Let $G^{\prime}$ be a (closed) subgroup of $G$ over $F$. The map of sets

$$
H^{1}\left(K, G^{\prime}\right) \rightarrow H^{1}(K, G)
$$

for every field extension $K / F$ yields the restriction map

$$
\text { res : } \operatorname{Inv}(G, H) \rightarrow \operatorname{Inv}\left(G^{\prime}, H\right) \text {. }
$$

Choose standard torsors $\pi: U \rightarrow U / G$ and $\pi^{\prime}: U \rightarrow U / G^{\prime}$ (for example, with $U=\mathbf{G L}_{n, F}$ as above). The pull-back of $\pi$ with respect to the natural morphism $\alpha: U / G^{\prime} \rightarrow U / G$ is the push-forward of $\pi^{\prime}$ via the inclusion $G^{\prime} \hookrightarrow G$. It follows that the diagram

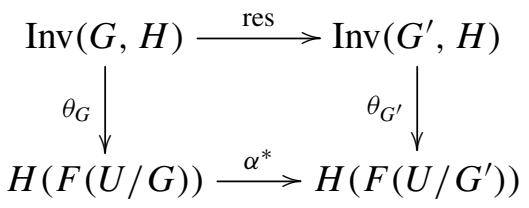

is commutative.

2c. The Brauer group invariants. Let $G$ be a smooth connected linear algebraic group over $F$. Every cohomological invariant of $G$ of degree 1 is constant by [Knus et al. 1998, Proposition 31.15]. In this section we study (degree 2) Br-invariants for the Brauer group functor $K \mapsto \operatorname{Br}(K)$. We assume that $G$ is reductive if $\operatorname{char}(F)>0$.

Lemma 2.3. For any field extension $K / F$ such that $F$ is algebraically closed in $K$, the natural map $\operatorname{Pic}(G) \rightarrow \operatorname{Pic}\left(G_{K}\right)$ is an isomorphism. 
Proof. We may assume that $G$ is reductive by factoring out the unipotent radical in the case that $F$ is perfect. There is an exact sequence (see [Colliot-Thélène 2004, Theorem 1.2])

$$
1 \rightarrow C \rightarrow G^{\prime} \rightarrow G \rightarrow 1
$$

with $C$ a torus and $G^{\prime}$ a reductive group with $\operatorname{Pic}\left(G_{L}^{\prime}\right)=0$ for any field extension $L / F$. Let $T$ be the factor group of $G^{\prime}$ by the semisimple part. The result follows from the exact sequence [Sansuc 1981, Proposition 6.10] (note that $G$ is reductive if $L$ is not perfect)

$$
\widehat{T}(L) \rightarrow \widehat{C}(L) \rightarrow \operatorname{Pic}\left(G_{L}\right) \rightarrow \operatorname{Pic}\left(G_{L}^{\prime}\right)=0
$$

with $L=F$ and $K$ since the groups $\widehat{T}(F)$ and $\widehat{C}(F)$ don't change when $F$ is replaced by $K$.

Since for any $G_{K}$-torsor $E \rightarrow \operatorname{Spec}(K)$ over a field extension $K / F$ one has [Sansuc 1981, Proposition 6.10] the exact sequence

$$
\operatorname{Pic}(E) \rightarrow \operatorname{Pic}\left(G_{K}\right) \stackrel{\delta}{\rightarrow} \operatorname{Br}(K) \stackrel{\varepsilon}{\rightarrow} \operatorname{Br}(E),
$$

we obtain the homomorphism

$$
v: \operatorname{Pic}(G) \rightarrow \operatorname{Inv}(G, \mathrm{Br}),
$$

which takes an element $\alpha \in \operatorname{Pic}(G)$ to the invariant that sends a $G$-torsor $E$ over a field extension $K / F$ to $\delta\left(\alpha_{K}\right)$. If $E$ is a trivial torsor, that is, $E(K) \neq \varnothing$, then $\varepsilon$ is injective and hence $\delta=0$. It follows that the invariant $v(\alpha)$ is normalized.

Theorem 2.4. Let $G$ be a smooth connected linear algebraic group over $F$. Assume that $G$ is reductive if $\operatorname{char}(F)>0$. Then the map $v: \operatorname{Pic}(G) \rightarrow \operatorname{Inv}(G, \operatorname{Br})_{\text {norm }}$ is an isomorphism.

Proof. Choose a standard classifying $G$-torsor $U \rightarrow U / G$. Write $K$ for the function field $F(U / G)$ and let $U_{\text {gen }}$ be the generic $G$-torsor over $K$. Consider the commutative diagram

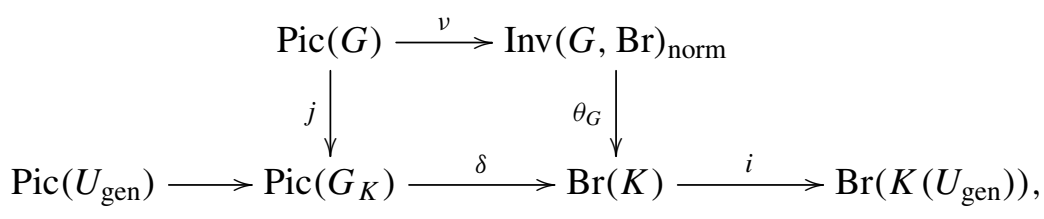

where the bottom sequence is (2-2) for the $G$-torsor $U_{\text {gen }} \rightarrow \operatorname{Spec}(K)$ followed by the injection $\operatorname{Br}\left(U_{\text {gen }}\right) \rightarrow \operatorname{Br}\left(K\left(U_{\text {gen }}\right)\right.$ ) (see [Milne 1980, Chapter IV, Corollary 2.6]), and the map $\theta_{G}$ is evaluation at the generic torsor $U_{\text {gen }}$ given in (2-1) and is injective by Theorem 2.2. Since the generic torsor is split over $K\left(U_{\text {gen }}\right)$, $\operatorname{Im}\left(\theta_{G}\right) \subset \operatorname{Ker}(i)=\operatorname{Im}(\delta)$. By Lemma 2.3, $j$ is an isomorphism, hence $v$ is surjective. 
Note that $U_{\text {gen }}$ is a localization of $U$, hence $\operatorname{Pic}\left(U_{\text {gen }}\right)=0$ as $\operatorname{Pic}(U)=0$. It follows that $v$ is injective.

An algebraic group $G$ over a field $F$ is called special if $H^{1}(K, G)=\{1\}$ for every field extension $K / F$, that is, all $G$-torsors over any field extension of $F$ are trivial. Corollary 2.5. If the group $G$ is special, then $\operatorname{Pic}(G)=0$.

\section{Invariants with values in $\mathbb{Q} / \mathbb{Z}(j)$}

In this section we find a description for the group of cohomological invariants with values in $\mathbb{Q} / \mathbb{Z}(j)$ by identifying the image of the embedding $\theta_{G}$ in (2-1).

Let $G$ be a linear algebraic group over a field $F$, let $H \subset G$ be a subgroup and let $E \rightarrow X$ be a $G$-torsor. Suppose that $G / H$ is affine. Consider a $G$-action on $E \times(G / H)$ by $\left(e, g^{\prime} H\right) g=\left(e g, g^{-1} g^{\prime} H\right)$. By [Milne 1980, Theorem I.2.23], the affine $G$-equivariant projection $E \times(G / H) \rightarrow E$ descends to an affine morphism $Y \rightarrow X$. The (trivial right) $H$-torsor $E \times G \rightarrow E \times(G / H)$ descends to an $H$-torsor $E \rightarrow Y$. We will write $E / H$ for $Y$.

Example 3.1. Let $G$ be a linear algebraic group over a field $F$ and let $E \rightarrow X$ be a $G$-torsor. Then for every $n>0, E^{n}:=E \times_{F} \cdots \times{ }_{F} E$ ( $n$ times) is a $G^{n}$-torsor over $X^{n}$. Viewing $G$ as the diagonal subgroup of $G^{n}$, we have the $G$-torsor $E^{n} \rightarrow E^{n} / G$.

3a. Balanced elements. Let $G$ be a linear algebraic group over a field $F$. We assume that $G$ is connected if $F$ is finite. Let $E \rightarrow X$ be a $G$-torsor such that $E(F) \neq \varnothing$. We write $p_{1}$ and $p_{2}$ for the two projections $E^{2} / G=\left(E \times{ }_{F} E\right) / G \rightarrow X$ (see Example 3.1).

Lemma 3.2. Let $K / F$ be a field extension and $x_{1}, x_{2} \in X(K)$. Then the $G$-torsors $E\left(x_{1}\right)$ and $E\left(x_{2}\right)$ over $K$ are isomorphic if and only if there is a point $y \in\left(E^{2} / G\right)(K)$ such that $p_{1}(y)=x_{1}$ and $p_{2}(y)=x_{2}$.

Proof. " $\Rightarrow$ ": By construction, we have $G$-equivariant morphisms $f_{i}: E\left(x_{i}\right) \rightarrow E$ for $i=1,2$. Choose an isomorphism $h: E\left(x_{1}\right) \stackrel{\sim}{\longrightarrow} E\left(x_{2}\right)$ of $G$-torsors over $K$. The morphism $\left(f_{1}, f_{2} h\right): E\left(x_{1}\right) \rightarrow E^{2}$ yields the required point $\operatorname{Spec} K=$ $E\left(x_{1}\right) / G \rightarrow E^{2} / G$.

" $\Leftarrow$ ": The pull-back of $E \rightarrow X$ with respect to any projection $E^{2} / G \rightarrow X$ coincides with the $G$-torsor $E^{2} \rightarrow E^{2} / G$, hence

$$
E\left(x_{1}\right)=x_{1}^{*}(E)=y^{*} p_{1}^{*}(E) \simeq y^{*}\left(E^{2}\right) \simeq y^{*} p_{2}^{*}(E)=x_{2}^{*}(E)=E\left(x_{2}\right) .
$$

Let $H$ be a (contravariant) functor from the category of schemes over $F$ to the category of abelian groups. We have the two maps $p_{i}^{*}: H(X) \rightarrow H\left(E^{2} / G\right), i=1,2$. An element $h \in H(X)$ is called balanced if $p_{1}^{*}(h)=p_{2}^{*}(h)$. We write $H(X)_{\text {bal }}$ for the subgroup of balanced elements in $H(X)$. In other words, $H(X)_{\text {bal }}=h_{0}\left(H\left(E^{\bullet} / G\right)\right)$ in the notation of Section A-IV. 
We can view $H$ as a (covariant) functor Fields $F \rightarrow$ Sets taking a field $K$ to $H(K):=H(\operatorname{Spec} K)$.

Lemma 3.3. Let $h \in H(X)_{\text {bal }}$ be a balanced element, $K / F$ a field extension and $I$ $a G$-torsor over $\operatorname{Spec}(K)$. Let $x \in X(K)$ be a point such that $E(x) \simeq I$. Then the element $x^{*}(h)$ in $H(K)$ does not depend on the choice of $x$.

Proof. Let $x_{1}, x_{2} \in X(K)$ be two points such that $E\left(x_{1}\right) \simeq E\left(x_{2}\right)$. By Lemma 3.2, there is a point $y \in\left(E^{2} / G\right)(K)$ such that $p_{1}(y)=x_{1}$ and $p_{2}(y)=x_{2}$. Therefore

$$
x_{1}^{*}(h)=y^{*}\left(p_{1}^{*}(h)\right)=y^{*}\left(p_{2}^{*}(h)\right)=x_{2}^{*}(h) .
$$

It follows from Lemma 3.3 that if the torsor $E \rightarrow X$ is classifying with $E(F) \neq \varnothing$, then every element $h \in H(X)_{\text {bal }}$ determines an $H$-invariant $i_{h}$ of $G$ as follows. Let $I$ be a $G$-torsor over a field extension $K / F$. We claim that there is a point $x \in X(K)$ such that $E(x) \simeq I$. If $K$ is infinite, this follows from the definition of the classifying $G$-torsor. If $K$ is finite then all $G$-torsors over $K$ are trivial by [Lang 1956], as $G$ is connected. Since $E(K) \neq \varnothing$, we can take for $x$ the image in $X(K)$ of any point in $E(K)$. Defining $i_{h}(E)=x^{*}(h) \in H(K)$, we have a group homomorphism

$$
H(X)_{\text {bal }} \rightarrow \operatorname{Inv}(G, H), \quad h \mapsto i_{h}
$$

3b. Cohomology with values in $\mathbb{Q} / \mathbb{Z}(j)$. For every integer $j \geq 0$, the coefficients $\mathbb{Q} / \mathbb{Z}(j)$ are defined as the direct sum over all prime integers $p$ of the objects $\mathbb{Q}_{p} / \mathbb{Z}_{p}(j)$ in the derived category of sheaves of abelian groups on the big étale site of $\operatorname{Spec} F$, where

$$
\mathbb{Q}_{p} / \mathbb{Z}_{p}(j)=\underset{n}{\operatorname{colim}} \mu_{p^{n}}^{\otimes j}
$$

if $p \neq \operatorname{char} F$, with $\mu_{p^{n}}$ the sheaf of $\left(p^{n}\right)$-th roots of unity, and

$$
\mathbb{Q}_{p} / \mathbb{Z}_{p}(j)=\underset{n}{\operatorname{colim}} W_{n} \Omega_{\log }^{j}[-j]
$$

if $p=$ char $F>0$, with $W_{n} \Omega_{\log }^{j}$ the sheaf of logarithmic de Rham-Witt differentials; see [Illusie 1979, I.5.7; Kahn 1996].

We write $H^{m}(X, \mathbb{Q} / \mathbb{Z}(j))$ for the étale cohomology of a scheme $X$ with values in $\mathbb{Q} / \mathbb{Z}(j)$. Then

$$
H^{m}(X, \mathbb{Q} / \mathbb{Z}(j))\{p\}=\operatorname{colim}_{n} H^{m}\left(X, \mu_{p^{n}}^{\otimes j}\right)
$$

if $p \neq \operatorname{char} F$ and

$$
H^{m}(X, \mathbb{Q} / \mathbb{Z}(j))\{p\}=\operatorname{colim}_{n} H^{m-j}\left(X, W_{n} \Omega_{\log }^{j}\right)
$$

if $p=$ char $F>0$. In the latter case, the group $W_{n} \Omega_{\log }^{j}(F)$ is canonically isomorphic to $K_{j}^{M}(F) / p^{n} K_{j}^{M}(F)$, where $K_{j}^{M}(F)$ is Milnor's $K$-group of $F$ (see 
[Bloch and Kato 1986, Corollary 2.8]), hence by [Izhboldin 1991; Garibaldi et al. 2003, Part II, Appendix A], $H^{s}\left(F, W_{n} \Omega_{\log }^{j}\right)$ is isomorphic to

$$
H^{s}\left(F, K_{j}^{M}\left(F_{\text {sep }}\right) / p^{n} K_{j}^{M}\left(F_{\text {sep }}\right)\right)= \begin{cases}K_{j}^{M}(F) / p^{n} K_{j}^{M}(F) & \text { if } s=0, \\ H^{2}\left(F, K_{j}^{M}\left(F_{\text {sep }}\right)\right)_{p^{n}} & \text { if } s=1, \\ 0 & \text { otherwise. }\end{cases}
$$

It follows that in the case $p=\operatorname{char} F>0$, we have

$$
H^{m}(F, \mathbb{Q} / \mathbb{Z}(j))\{p\}= \begin{cases}K_{j}^{M}(F) \otimes\left(\mathbb{Q}_{p} / \mathbb{Z}_{p}\right) & \text { if } m=j, \\ H^{2}\left(F, K_{j}^{M}\left(F_{\text {sep }}\right)\right)\{p\} & \text { if } m=j+1, \\ 0 & \text { otherwise. }\end{cases}
$$

The motivic complexes $\mathbb{Z}(j)$, for $j=0,1,2$, of étale sheaves on a smooth scheme $X$ were defined by S. Lichtenbaum $[1987 ; 1990]$. We write $H^{*}(X, \mathbb{Z}(j))$ for the étale (hyper)cohomology groups of $X$ with values in $\mathbb{Z}(j)$.

The complex $\mathbb{Z}(0)$ is equal to the constant sheaf $\mathbb{Z}$ and $\mathbb{Z}(1)=\mathbb{G}_{m, X}[-1]$, thus $H^{n}(X, \mathbb{Z}(1))=H^{n-1}\left(X, \mathbb{G}_{m, X}\right)$. In particular, $H^{3}(X, \mathbb{Z}(1))=\operatorname{Br}(X)$, the cohomological Brauer group of $X$. The complex $\mathbb{Z}(2)$ is concentrated in degrees 1 and 2 and there is a product map $\mathbb{Z}(1) \otimes^{L} \mathbb{Z}(1) \rightarrow \mathbb{Z}(2)$; see [Lichtenbaum 1987, Proposition 2.5].

The exact triangle in the derived category of étale sheaves

$$
\mathbb{Z}(j) \rightarrow \mathbb{Q} \otimes \mathbb{Z}(j) \rightarrow \mathbb{Q} / \mathbb{Z}(j) \rightarrow \mathbb{Z}(j)[1]
$$

yields the connecting homomorphism

$$
H^{i}(X, \mathbb{Q} / \mathbb{Z}(j)) \rightarrow H^{i+1}(X, \mathbb{Z}(j)),
$$

which is an isomorphism if $X=\operatorname{Spec}(F)$ for a field $F$ and $i>j$ [Kahn 1993, Lemme 1.1].

Write $\mathscr{H}^{n}(\mathbb{Q} / \mathbb{Z}(j))$ for the Zariski sheaf on a smooth scheme $X$ associated to the presheaf $U \mapsto H^{n}(U, \mathbb{Q} / \mathbb{Z}(j))$ of étale cohomology groups.

Let $G$ be a linear algebraic group over $F$. We assume that $G$ is connected if $F$ is a finite field and write $\operatorname{Inv}^{n}(G, \mathbb{Q} / \mathbb{Z}(j))$ for the group of degree $n$ invariants of $G$ for the functor $K \mapsto H^{n}(K, \mathbb{Q} / \mathbb{Z}(j))$. Note that Property 2.1 holds for this functor by [Garibaldi et al. 2003, Part 2, Proposition A.9].

Choose a classifying $G$-torsor $E \rightarrow X$ with $E$ a $G$-rational variety such that $E(F) \neq \varnothing$. Applying the construction given in Section 3 a to the functor $U \mapsto$ $H_{\text {Zar }}^{0}\left(U, \mathscr{H}^{n}(\mathbb{Q} / \mathbb{Z}(j))\right)$, we get a homomorphism

$$
\varphi: H_{\mathrm{Zar}}^{0}\left(X, \mathscr{H}^{n}(\mathbb{Q} / \mathbb{Z}(j))\right)_{\mathrm{bal}} \rightarrow \operatorname{Inv}^{n}(G, \mathbb{Q} / \mathbb{Z}(j)) .
$$

Theorem 3.4. Let $G$ be a smooth linear algebraic group over a field $F$. We assume that $G$ is connected if $F$ is a finite field. Let $E \rightarrow X$ be a classifying $G$-torsor 
with $E$ a $G$-rational variety such that $E(F) \neq \varnothing$. Then the homomorphism $\varphi$ is an isomorphism.

Proof. Let $E_{\text {gen }} \rightarrow F(X)$ be the generic fiber of the classifying $G$-torsor $E \rightarrow X$. Note that since the pull-back of $E \rightarrow X$ with respect to any of the two projections $E^{2} / G \rightarrow X$ coincides with the $G$-torsor $E^{2} \rightarrow E^{2} / G$, the pull-backs of the generic $G$-torsor $E_{\mathrm{gen}} \rightarrow \operatorname{Spec} F(X)$ with respect to the two morphisms Spec $F\left(E^{2} / G\right) \rightarrow$ Spec $F(X)$ induced by the projections are isomorphic. It follows that for every invariant $i \in \operatorname{Inv}\left(G, H^{*}(\mathbb{Q} / \mathbb{Z}(j))\right)$ we have

$$
p_{1}^{*}\left(i\left(E_{\text {gen }}\right)\right)=i\left(p_{1}^{*}\left(E_{\text {gen }}\right)\right)=i\left(p_{2}^{*}\left(E_{\text {gen }}\right)\right)=p_{2}^{*}\left(i\left(E_{\text {gen }}\right)\right)
$$

in $H^{*}\left(F\left(E^{2} / G\right), \mathbb{Q} / \mathbb{Z}(j)\right)$, that is, $i\left(E_{\text {gen }}\right) \in H^{*}(F(X), \mathbb{Q} / \mathbb{Z}(j))_{\text {bal. }}$. By Proposition A.9, $\partial_{x}(h)=0$ for every point $x \in X$ of codimension 1 , hence

$$
\theta_{G}(i)=i\left(E_{\text {gen }}\right) \in H_{\text {Zar }}^{0}\left(X, \mathscr{H}^{n}(\mathbb{Q} / \mathbb{Z}(j))\right)_{\text {bal }}
$$

by Proposition A.10. By Theorem 2.2, $\theta_{G}$ is injective and by construction, the composition $\theta_{G} \circ \varphi$ is the identity. It follows that $\varphi$ is an isomorphism.

Write $\bar{H}_{\mathrm{Zar}}^{0}\left(X, \mathcal{H}^{n}(\mathbb{Q} / \mathbb{Z}(j))\right)$ for the factor group of $H_{\mathrm{Zar}}^{0}\left(X, \mathscr{H}^{n}(\mathbb{Q} / \mathbb{Z}(j))\right)$ by the natural image of $H^{n}(F, \mathbb{Q} / \mathbb{Z}(j))$.

Corollary 3.5. The isomorphism $\varphi$ yields an isomorphism

$$
\bar{H}_{\text {Zar }}^{0}\left(X, \mathscr{H}^{n}(\mathbb{Q} / \mathbb{Z}(j))\right)_{\text {bal }} \stackrel{\sim}{\longrightarrow} \operatorname{Inv}^{n}(G, \mathbb{Q} / \mathbb{Z}(j))_{\text {norm }} .
$$

\section{Degree 3 invariants of algebraic tori}

In this section we prove the main theorem that describes degree 3 invariants of an algebraic torus with values in $\mathbb{Q} / \mathbb{Z}(2)$.

4a. Algebraic tori. Let $F$ be a field and $\Gamma=\operatorname{Gal}\left(F_{\text {sep }} / F\right)$ the absolute Galois group of $F$. An algebraic torus of dimension $n$ over $F$ is an algebraic group $T$ such that $T_{\text {sep }}$ is isomorphic to the product of $n$ copies of the multiplicative group $\mathbb{G}_{m}$; see [Colliot-Thélène and Sansuc 1977; Voskresenskiü 1998]. For an algebraic torus $T$ over a field $F$, we write $\widehat{T}_{\text {sep }}$ for the $\Gamma$-module of characters $\operatorname{Hom}\left(T_{\text {sep }}, \mathbb{G}_{m}\right)$. The group $\widehat{T}_{\text {sep }}$ is a $\Gamma$-lattice, that is, a free abelian group of finite rank with a continuous $\Gamma$-action. The contravariant functor $T \mapsto \widehat{T}_{\text {sep }}$ is an antiequivalence between the category of algebraic tori and the category of $\Gamma$-lattices: the torus $T$ and the group $T(F)$ can be reconstructed from the lattice $\widehat{T}_{\text {sep }}$ by the formulas

$$
\begin{aligned}
T & =\operatorname{Spec}\left(F_{\text {sep }}\left[\widehat{T}_{\text {sep }}\right]^{\Gamma}\right), \\
T(F) & =\operatorname{Hom}_{\Gamma}\left(\widehat{T}_{\text {sep }}, F_{\text {sep }}^{\times}\right)=\left(\widehat{T}_{\text {sep }}^{\circ} \otimes F_{\text {sep }}^{\times}\right)^{\Gamma},
\end{aligned}
$$

where $\widehat{T}_{\text {sep }}^{\circ}=\operatorname{Hom}\left(\widehat{T}_{\text {sep }}, \mathbb{Z}\right)$. 
We write $\widehat{T}$ for the character group $\operatorname{Hom}_{F}\left(T, \mathbb{G}_{m}\right)=\left(\widehat{T}_{\text {sep }}\right)^{\Gamma}$ and $T^{\circ}$ for the dual torus having character lattice $\widehat{T}_{\text {sep }}^{\circ}$.

A torus $T$ is called quasisplit if $T$ is isomorphic to the group of invertible elements of an étale $F$-algebra, or equivalently, the $\Gamma$-lattice $\widehat{T}_{\text {sep }}$ is permutation, that is, $\widehat{T}_{\text {sep }}$ has a $\Gamma$-invariant $\mathbb{Z}$-basis. An invertible torus is a direct factor of a quasisplit torus.

A torus $T$ is called flasque or coflasque if $H^{1}\left(L, \widehat{T}_{\text {sep }}^{\circ}\right)=0$ or $H^{1}\left(L, \widehat{T}_{\mathrm{sep}}\right)=0$, respectively, for every finite field extension $L / F$. A flasque resolution of a torus $S$ is an exact sequence of tori $1 \rightarrow T \rightarrow P \rightarrow S \rightarrow 1$ with $T$ flasque and $P$ quasisplit. By [Colliot-Thélène and Sansuc 1977, §4; Voskresenskiǔ 1998, §4.7], the torus $T$ in the flasque resolution is invertible if and only if $S$ is a direct factor of a rational torus.

4b. Products. Let $T$ be a torus over $F$ and let $\widehat{T}(i)$ denote the complex $\widehat{T}_{\text {sep }} \otimes \mathbb{Z}(i)$ of étale sheaves over $F$ for $i=0,1,2$. Thus, $\widehat{T}(0)=\widehat{T}_{\text {sep }}$ and

$$
\widehat{T}(1)=\left(\widehat{T}_{\text {sep }} \otimes F_{\text {sep }}^{\times}\right)[-1]=T^{\circ}\left(F_{\text {sep }}\right)[-1] .
$$

Let $S$ and $T$ be algebraic tori over $F$ and let $i$ and $j$ be nonnegative integers with $i+j \leq 2$. For any smooth variety $X$ over $F$, we have the product map

$$
\left(\widehat{S}_{\text {sep }} \otimes \widehat{T}_{\text {sep }}\right)^{\Gamma} \otimes H^{p}\left(X, \widehat{S}^{\circ}(i)\right) \otimes H^{q}\left(X, \widehat{T}^{\circ}(j)\right) \rightarrow H^{p+q}(X, \mathbb{Z}(i+j))
$$

taking $a \otimes b \otimes c$ to $a \cup b \cup c$, via the canonical pairings between $\widehat{S}_{\text {sep }}$ and $\widehat{S}_{\text {sep }}^{\circ}, \widehat{T}_{\text {sep }}$ and $\widehat{T}_{\text {sep }}^{\circ}$, and the product map $\mathbb{Z}(i) \otimes^{L} \mathbb{Z}(j) \rightarrow \mathbb{Z}(i+j)$.

Recall that there is an isomorphism $H^{n}(F, \mathbb{Z}(k)) \simeq H^{n-1}(F, \mathbb{Q} / \mathbb{Z}(k))$ for $n>k$. In particular, we have the cup-product map

$$
\left(\widehat{S}_{\text {sep }} \otimes \widehat{T}_{\text {sep }}\right)^{\Gamma} \otimes H^{p}(F, S) \otimes H^{q}(F, T) \rightarrow H^{3}(F, \mathbb{Q} / \mathbb{Z}(2))
$$

if $p+q=2$.

If $S=T^{\circ}$ is the dual torus, then $\left(\widehat{S}_{\text {sep }} \otimes \widehat{T}_{\text {sep }}\right)^{\Gamma}=\operatorname{End}_{\Gamma}\left(\widehat{T}_{\text {sep }}\right)$ contains the canonical element $1_{T}$. We then have the product map

$$
H^{p}(X, \widehat{T}(i)) \otimes H^{q}\left(X, \widehat{T}^{\circ}(j)\right) \rightarrow H^{p+q}(X, \mathbb{Z}(i+j))
$$

and in particular, the product maps

$$
\begin{aligned}
H^{1}\left(F, \widehat{T}_{\mathrm{sep}}\right) \otimes H^{1}(F, T) & \rightarrow H^{2}(F, \mathbb{Q} / \mathbb{Z}(1))=\operatorname{Br}(F), \\
H^{1}\left(F, T^{\circ}\right) \otimes H^{1}(F, T) & \rightarrow H^{3}(F, \mathbb{Q} / \mathbb{Z}(2)), \\
H^{2}\left(F, T^{\circ}\right) \otimes H^{0}(F, T) & \rightarrow H^{3}(F, \mathbb{Q} / \mathbb{Z}(2)),
\end{aligned}
$$

taking $a \otimes b$ to $1_{T} \cup a \cup b$ and applying (4-2).

As $T$ is a commutative group, the set $H^{1}(K, T)$ is an abelian group. An invariant $i \in \operatorname{Inv}(T, H)$ for a functor $H$ is called linear if $i_{K}: H^{1}(K, T) \rightarrow H(K)$ is a group 
homomorphism for every $K / F$. In the next section we will see that a normalized degree 3 invariant of a torus need not be linear.

4c. Main theorem. Let $T$ be a torus over $F$ and choose a standard classifying $T$-torsor $U \rightarrow U / T$ such that the codimension of $V \backslash U$ in $V$ is at least 3. Such a torsor exists by [Edidin and Graham 1998, Lemma 9].

By [Sansuc 1981, Proposition 6.10], there is an exact sequence

$$
F_{\text {sep }}[U]^{\times} / F_{\text {sep }}^{\times} \rightarrow \widehat{T}_{\text {sep }} \rightarrow \operatorname{Pic}\left((U / T)_{\text {sep }}\right) \rightarrow \operatorname{Pic}\left(U_{\text {sep }}\right) .
$$

The codimension assumption implies that the side terms are trivial, hence the map $\widehat{T}_{\text {sep }} \rightarrow \operatorname{Pic}\left((U / T)_{\text {sep }}\right)$ is an isomorphism. It follows that the classifying $T$-torsor $U \rightarrow U / T$ is universal in the sense of [Colliot-Thélène and Sansuc 1987a].

Write $K_{*}(F)$ for the (Quillen) $K$-groups of $F$ and $\mathcal{K}_{*}$ for the Zariski sheaf associated to the presheaf $U \mapsto K_{*}(U)$. Then the groups $H_{\mathrm{Zar}}^{n}\left(U / T, \mathscr{K}_{2}\right)$ are independent of the choice of the classifying torsor; see [Edidin and Graham 1998]. So we write $H_{\mathrm{Zar}}^{n}\left(B T, \mathscr{K}_{2}\right)$ for this group (see Section A-IV). As $T_{\text {sep }}$ is a split torus, by the Künneth formula (see Example A.5),

$$
H_{\mathrm{Zar}}^{n}\left(B T_{\text {sep }}, \mathscr{K}_{2}\right)= \begin{cases}K_{2}\left(F_{\text {sep }}\right) & \text { if } n=0, \\ \operatorname{Pic}\left((U / T)_{\text {sep }}\right) \otimes F_{\text {sep }}^{\times}=\widehat{T}_{\text {sep }} \otimes F_{\text {sep }}^{\times}=T^{\circ}\left(F_{\text {sep }}\right) & \text { if } n=1, \\ \mathrm{CH}^{2}\left((U / T)_{\text {sep }}\right)=S^{2}\left(\widehat{T}_{\text {sep }}\right) & \text { if } n=2 .\end{cases}
$$

Applying the calculation of the $\mathscr{K}$-cohomology groups to the standard classifying $T$-torsor $U^{i} \rightarrow U^{i} / T$ for every $i>0$ instead of $U \rightarrow U / T$, by Proposition B.3, we have the exact sequence

$$
\begin{aligned}
0 \rightarrow H^{1}\left(F, T^{\circ}\right) \stackrel{\alpha}{\rightarrow} \bar{H}^{4}\left(U^{i} / T, \mathbb{Z}(2)\right) & \\
& \rightarrow \bar{H}^{4}\left(\left(U^{i} / T\right)_{\mathrm{sep}}, \mathbb{Z}(2)\right)^{\Gamma} \rightarrow H^{2}\left(F, T^{\circ}\right),
\end{aligned}
$$

where $\bar{H}^{4}\left(U^{i} / T, \mathbb{Z}(2)\right)$ is the factor group of $H^{4}\left(U^{i} / T, \mathbb{Z}(2)\right)$ by $H^{4}(F, \mathbb{Z}(2))$, the map $\alpha$ is given by $\alpha(a)=q^{*}(a) \cup\left[U^{i}\right]$ with $q: U^{i} / T \rightarrow \operatorname{Spec} F$ the structure morphism, $\left[U^{i}\right]$ the class of the $T$-torsor $U^{i} \rightarrow U^{i} / T$ in $H^{1}\left(U^{i} / T, T\right)$, and the cup-product is taken for the pairing (B-6).

Taking the sequences (4-7) for all $i$ (see Section A-IV), we get the exact sequence of cosimplicial groups

$$
0 \rightarrow H^{1}\left(F, T^{\circ}\right) \stackrel{\alpha}{\rightarrow} \bar{H}^{4}\left(U^{\bullet} / T, \mathbb{Z}(2)\right) \rightarrow \bar{H}^{4}\left(\left(U^{\bullet} / T\right)_{\mathrm{sep}}, \mathbb{Z}(2)\right)^{\Gamma} \rightarrow H^{2}\left(F, T^{\circ}\right) .
$$

The first and the last cosimplicial groups in the sequence are constant, hence by Lemma A.2, the sequence

$0 \rightarrow H^{1}\left(F, T^{\circ}\right) \stackrel{\alpha}{\rightarrow} \bar{H}^{4}(U / T, \mathbb{Z}(2))_{\text {bal }}$

$$
\rightarrow \bar{H}^{4}\left((U / T)_{\mathrm{sep}}, \mathbb{Z}(2)\right)_{\mathrm{bal}}^{\Gamma} \rightarrow H^{2}\left(F, T^{\circ}\right)
$$


is exact as $h_{0}\left(\bar{H}^{4}\left(U^{\bullet} / T, \mathbb{Z}(2)\right)\right)=H^{4}(U / T, \mathbb{Z}(2))_{\text {bal }}$.

The following theorem was proved by B. Kahn [1996, Theorem 1.1]:

Theorem 4.1. Let $X$ be a smooth variety over $F$. Then there is an exact sequence

$$
0 \rightarrow \mathrm{CH}^{2}(X) \rightarrow H^{4}(X, \mathbb{Z}(2)) \rightarrow H_{\mathrm{Zar}}^{0}\left(X, \mathscr{H}^{3}(\mathbb{Q} / \mathbb{Z}(2))\right) \rightarrow 0 .
$$

By Theorem 4.1, there is an exact sequence of cosimplicial groups

$$
0 \rightarrow \mathrm{CH}^{2}\left(U^{\bullet} / T\right) \rightarrow \bar{H}^{4}\left(U^{\bullet} / T, \mathbb{Z}(2)\right) \rightarrow \bar{H}_{\mathrm{Zar}}^{0}\left(U^{\bullet} / T, \mathscr{H}^{3}(\mathbb{Q} / \mathbb{Z}(2))\right) \rightarrow 0 .
$$

As the functor $\mathrm{CH}^{2}$ is homotopy invariant, by Lemma A.4, the first group in the sequence is constant. In view of Lemma A.2, and following the notation for the $\mathscr{K}$-cohomology, the sequence

$$
0 \rightarrow \mathrm{CH}^{2}(B T) \rightarrow \bar{H}^{4}(U / T, \mathbb{Z}(2))_{\text {bal }} \rightarrow \bar{H}_{\mathrm{Zar}}^{0}\left(U / T, \mathscr{H}^{3}(\mathbb{Q} / \mathbb{Z}(2))\right)_{\mathrm{bal}} \rightarrow 0
$$

is exact. By Corollary 3.5, the last group in the sequence is canonically isomorphic to $\operatorname{Inv}\left(T, H^{3}(\mathbb{Q} / \mathbb{Z}(2))\right)_{\text {norm }}$.

As the torus $T_{\text {sep }}$ is split, all the invariants of $T_{\text {sep }}$ are trivial hence the sequence (4-9) over $F_{\text {sep }}$ yields an isomorphism

$$
\bar{H}^{4}\left((U / T)_{\mathrm{sep}}, \mathbb{Z}(2)\right)_{\mathrm{bal}} \simeq \mathrm{CH}^{2}\left(B T_{\mathrm{sep}}\right) \simeq S^{2}\left(\widehat{T}_{\mathrm{sep}}\right) .
$$

Combining (4-8), (4-9) and (4-10), we get the following diagram with an exact row and column:

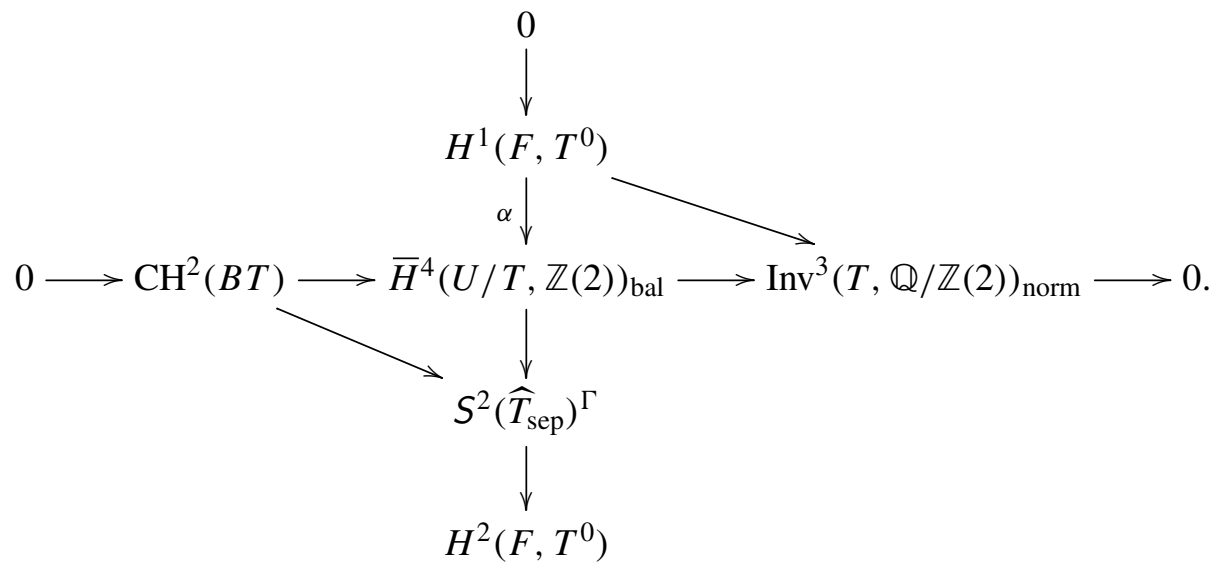

Write Dec $=\operatorname{Dec}\left(\widehat{T}_{\mathrm{sep}}\right)$ for the subgroup of decomposable elements in $S^{2}\left(\widehat{T}_{\mathrm{sep}}\right)^{\Gamma}$ (see Section A-II).

Lemma 4.2. The image of the homomorphism

$$
\mathrm{CH}^{2}(B T) \rightarrow \mathrm{CH}^{2}\left(B T_{\mathrm{sep}}\right)^{\Gamma}=S^{2}\left(\widehat{T}_{\mathrm{sep}}\right)^{\Gamma}
$$

in the diagram coincides with Dec. 
Proof. Consider the Grothendieck ring $K_{0}(B T)$ of the category of $T$-equivariant vector bundles over $\operatorname{Spec}(F)$, or equivalently, of the category of finite dimensional linear representations of $T$. If $T$ is split, every linear representation of $T$ is a direct sum of one-dimensional representations. Therefore, there is an isomorphism between the group ring $\mathbb{Z}[\widehat{T}]$ of all formal finite sums $\sum_{x \in \widehat{T}} a_{x} e^{x}$ and $K_{0}(B T)$, taking $e^{x}$ with $x \in \widehat{T}$ to the class of the 1-dimensional representation given by $x$. In general, for every torus $T$, we have $K_{0}\left(B T_{\text {sep }}\right)=\mathbb{Z}\left[\widehat{T}_{\text {sep }}\right]$ and $K_{0}(B T)=\mathbb{Z}\left[\widehat{T}_{\text {sep }}\right]^{\Gamma}=K_{0}\left(B T_{\text {sep }}\right)^{\Gamma}$; see [Merkurjev and Panin 1997, page 136]. The group $\mathbb{Z}\left[\widehat{T}_{\text {sep }}\right]^{\Gamma}$ is generated by the sums $\sum_{i=1}^{n} e^{\gamma_{i} x}$, where $\gamma_{1}, \gamma_{2}, \ldots, \gamma_{n}$ are representatives of the left cosets of an arbitrary open subgroup $\Gamma^{\prime}$ in $\Gamma$ and $x \in\left(\widehat{T}_{\text {sep }}\right)^{\Gamma^{\prime}}$.

The equivariant Chern classes were defined in [Edidin and Graham 1998, §2.4]. The first Chern class $c_{1}: K_{0}\left(B T_{\text {sep }}\right) \rightarrow \mathrm{CH}^{1}\left(B T_{\text {sep }}\right)=\widehat{T}_{\text {sep }}$ takes $e^{x}$ to $x$. In the diagram

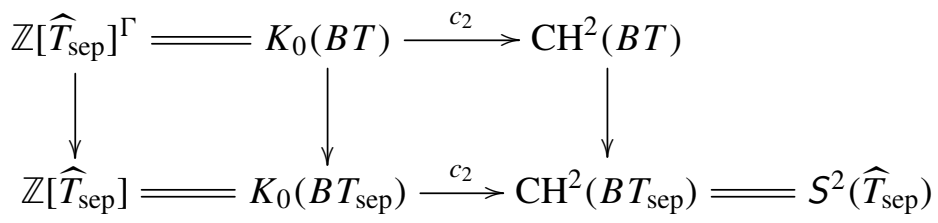

the second Chern class maps $c_{2}$ are surjective by [Esnault et al. 1998, Lemma C.3]. It follows from the formula $c_{2}(a+b)=c_{2}(a)+c_{1}(a) c_{1}(b)+c_{2}(b)$ that the composition

$$
\mathbb{Z}\left[\widehat{T}_{\mathrm{sep}}\right]^{\Gamma}=K_{0}(B T) \rightarrow K_{0}\left(B T_{\mathrm{sep}}\right) \stackrel{c_{2}}{\rightarrow} \mathrm{CH}^{2}\left(B T_{\mathrm{sep}}\right)=S^{2}\left(\widehat{T}_{\mathrm{sep}}\right) \rightarrow S^{2}\left(\widehat{T}_{\mathrm{sep}}\right) /(\widehat{T})^{2}
$$

is a homomorphism and its image is generated by the elements (see Section A-II)

$$
c_{2}\left(\sum_{i=1}^{n} e^{\gamma_{i} x}\right)=\sum_{i<j}\left(\gamma_{i} x\right)\left(\gamma_{j} x\right)=\operatorname{Qtr}(x) .
$$

By the restriction-corestriction argument, the kernel of the homomorphism

$$
\mathrm{CH}^{2}(B T) \rightarrow \mathrm{CH}^{2}\left(B T_{\mathrm{sep}}\right)^{\Gamma}=S^{2}\left(\widehat{T}_{\mathrm{sep}}\right)^{\Gamma}
$$

coincides with the torsion subgroup $\mathrm{CH}^{2}(B T)_{\text {tors }}$ in $\mathrm{CH}^{2}(B T)$.

The following theorem describes degree 3 invariants of an algebraic torus with values in $\mathbb{Q} / \mathbb{Z}(2)$ :

Theorem 4.3. Let $T$ be an algebraic torus a field $F$. Then there is an exact sequence $0 \rightarrow \mathrm{CH}^{2}(B T)_{\text {tors }} \rightarrow H^{1}\left(F, T^{0}\right) \stackrel{\alpha}{\rightarrow} \operatorname{Inv}^{3}(T, \mathbb{Q} / \mathbb{Z}(2))_{\text {norm }}$

$$
\rightarrow S^{2}\left(\widehat{T}_{\mathrm{sep}}\right)^{\Gamma} / \operatorname{Dec} \rightarrow H^{2}\left(F, T^{0}\right) .
$$

The homomorphism $\alpha$ is given by $\alpha(a)(b)=a_{K} \cup b$ for every $a \in H^{1}\left(F, T^{0}\right)$ and $b \in H^{1}(K, T)$ and every field extension $K / F$, where the cup-product is defined in (4-5). 
Proof. The exactness of the sequence follows from the diagram before Lemma 4.2. It remains to describe the map $\alpha$. Take an $a \in H^{1}\left(F, T^{0}\right)$ and consider the invariant $i$ defined by $i(b)=a_{K} \cup b$, where the cup-product is given by (4-5). We need to prove that $i=\alpha(a)$. Choose a standard classifying $T$-torsor $U \rightarrow U / T$ and set $K=F(U / T)$. Let $U_{\text {gen }}$ be the generic fiber of the classifying torsor. By Theorem 2.2, it suffices to show that $i\left(U_{\text {gen }}\right)=\alpha(a)\left(U_{\text {gen }}\right)$ over $K$. This follows from the description of the map $\alpha$ in the exact sequence (4-7).

Remark 4.4. In a similar (and much simpler) fashion one can describe degree 2 invariants of an algebraic torus $T$ with values in $\mathbb{Q} / \mathbb{Z}(1)$, that is, invariants with values in the Brauer group by computing the étale motivic cohomology group $H^{3}(U / T, \mathbb{Z}(1))=H^{2}\left(U / T, \mathbb{G}_{m}\right)=\operatorname{Br}(U / T)$ for a standard classifying $T$-torsor $U \rightarrow U / T$. One establishes canonical isomorphisms

$$
H^{1}\left(F, \widehat{T}_{\mathrm{sep}}\right) \simeq \bar{H}^{3}(U / T, \mathbb{Z}(1))_{\mathrm{bal}} \simeq \operatorname{Inv}^{2}(T, \mathbb{Q} / \mathbb{Z}(1))_{\mathrm{norm}}=\operatorname{Inv}(T, \mathrm{Br})_{\mathrm{norm}} .
$$

The composition takes an element $a \in H^{1}\left(F, \widehat{T}_{\text {sep }}\right)$ to the invariant $b \mapsto a_{K} \cup b$ for $b \in H^{1}(K, T)$ and a field extension $K / F$. This description shows that every normalized $\mathrm{Br}$-invariant of $T$ is linear.

4d. Torsion in $\mathbf{C H}^{2}(\boldsymbol{B T})$. We investigate the group $\mathrm{CH}^{2}(B T)_{\text {tors }}$, the first term of the exact sequence in Theorem 4.3.

Let $S$ be an algebraic torus over $F$. Using the Gersten resolution, [Quillen 1973, Proposition 5.8] we identify the group $H^{0}\left(S_{\text {sep }}, \mathscr{K}_{2}\right)$ with a subgroup in $K_{2}\left(F_{\text {sep }}(S)\right)$. Set $\bar{H}^{0}\left(S_{\text {sep }}, \mathscr{K}_{2}\right):=H^{0}\left(S_{\text {sep }}, \mathscr{K}_{2}\right) / K_{2}\left(F_{\text {sep }}\right)$. By [Garibaldi et al. 2003, Part 2, §5.7], we have an exact sequence

$$
0 \rightarrow \widehat{S}_{\text {sep }} \otimes F_{\text {sep }}^{\times} \rightarrow \bar{H}^{0}\left(S_{\text {sep }}, \mathscr{K}_{2}\right) \stackrel{\lambda}{\rightarrow} \bigwedge^{2} \widehat{S}_{\text {sep }} \rightarrow 0
$$

of $\Gamma$-modules, where $\lambda\left(\left\{e^{x}, e^{y}\right\}\right)=x \wedge y$ for $x, y \in \widehat{S}_{\text {sep }}$.

Consider the $\Gamma$-homomorphism

$$
\begin{aligned}
\gamma: \wedge^{2} \widehat{S}_{\mathrm{sep}} & \rightarrow \bar{H}^{0}\left(S_{\mathrm{sep}}, \mathscr{K}_{2}\right) \\
x \wedge y & \mapsto\left\{e^{x}, e^{y}\right\}-\left\{e^{y}, e^{x}\right\} .
\end{aligned}
$$

We have $\lambda \circ \gamma=2 \cdot I d$, hence the connecting homomorphism

$$
\partial: H^{i}\left(F, \bigwedge^{2} \widehat{S}_{\text {sep }}\right) \rightarrow H^{i+1}\left(F, \widehat{S}_{\text {sep }} \otimes F_{\text {sep }}^{\times}\right)
$$

satisfies $2 \partial=0$.

Lemma 4.5. If $S$ is an invertible torus, then the sequence of $\Gamma$-modules (4-11) is split. 
Proof. Suppose first that $S$ is quasisplit. Let $\left\{x_{1}, x_{2}, \ldots, x_{m}\right\}$ be a permutation basis

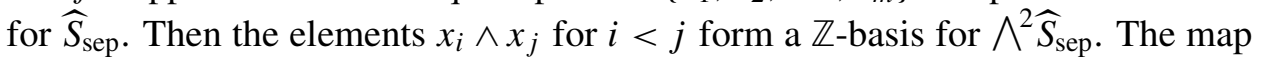
$\wedge^{2} \widehat{S}_{\text {sep }} \rightarrow \bar{H}^{0}\left(S_{\text {sep }}, \mathscr{K}_{2}\right)$, taking $x_{i} \wedge x_{j}$ to $\left\{e^{x_{i}}, e^{x_{j}}\right\}$ is a splitting for $\gamma$.

In general, find a torus $S^{\prime}$ such that $S \times S^{\prime}$ is quasisplit. The desired splitting is the composition

$$
\bigwedge^{2} \widehat{S}_{\text {sep }} \rightarrow \bigwedge^{2} S_{\text {sep }} \times S_{\text {sep }}^{\prime} \stackrel{\alpha}{\rightarrow} \bar{H}^{0}\left(S_{\text {sep }} \times S_{\text {sep }}^{\prime}, \mathscr{K _ { 2 }}\right) \stackrel{\beta}{\rightarrow} \bar{H}^{0}\left(S_{\text {sep }}, \mathscr{K}_{2}\right),
$$

where $\alpha$ is a splitting for the torus $S \times S^{\prime}$ and $\beta$ is the pull-back map for the canonical inclusion $S \hookrightarrow S \times S^{\prime}$.

Let

$$
1 \rightarrow T \rightarrow P \rightarrow Q \rightarrow 1
$$

be a coflasque resolution of $T$, that is, $P$ is a quasisplit torus and $Q$ is a coflasque torus; see [Colliot-Thélène and Sansuc 1977]. The torus $P$ is an open set in the affine space of a separable $F$-algebra on which $T$ acts linearly. Hence $P \rightarrow Q$ is a standard classifying $T$-torsor. By Theorem 2.2, the natural map

$$
\theta_{T}: \operatorname{Inv}^{3}(T, \mathbb{Q} / \mathbb{Z}(2)) \rightarrow H^{3}(F(Q), \mathbb{Q} / \mathbb{Z}(2))
$$

is injective.

Consider the spectral sequence (B-10) for the variety $X=Q$. By [Garibaldi et al. 2003, Part 2, Corollary 5.6], we have $H^{1}\left(Q_{\text {sep }}, \mathscr{K}_{2}\right)=0$. In view of Proposition B.4, we have an injective homomorphism

$$
\beta: H^{2}\left(F, \bar{H}^{0}\left(Q_{\text {sep }}, \mathscr{K}_{2}\right)\right) \rightarrow \bar{H}^{4}(Q, \mathbb{Z}(2))
$$

such that the composition of $\beta$ with the homomorphism

$$
H^{2}\left(F, Q^{\circ}\right) \rightarrow H^{2}\left(F, \bar{H}^{0}\left(Q_{\text {sep }}, \mathscr{K}_{2}\right)\right)
$$

is given by the cup-product with the class of the identity in $H^{0}(Q, Q)$.

Lemma 4.6. For a coflasque torus $Q$, the group $\mathrm{CH}^{2}(Q)$ is trivial.

Proof. By [Merkurjev and Panin 1997, Theorem 9.1], for every torus $Q$, the Grothendieck group $K_{0}(Q)$ is generated by the classes of the sheaves $i_{*}(P)$, where $P$ is an invertible sheaf on $Q_{L}, L / F$ a finite separable field extension and $i: Q_{L} \rightarrow Q$ is the natural morphism. By definition of a coflasque torus,

$$
\operatorname{Pic}\left(Q_{L}\right)=H^{1}\left(L, \widehat{Q}_{\mathrm{sep}}\right)=0 .
$$

It follows that every invertible sheaf on $Q_{L}$ is trivial, hence $K_{0}(Q)=\mathbb{Z} \cdot 1$. Since the group $\mathrm{CH}^{2}(Q)$ is generated by the second Chern classes of vector bundles on $Q$ [Esnault et al. 1998, Lemma C.3], we have $\mathrm{CH}^{2}(Q)=0$. 
It follows from Proposition A.10, Theorem 4.1, and Lemma 4.6 that the homomorphism

$$
\kappa: \bar{H}^{4}(Q, \mathbb{Z}(2)) \rightarrow \bar{H}^{4}(F(Q), \mathbb{Z}(2))=\bar{H}^{3}(F(Q), \mathbb{Q} / \mathbb{Z}(2))
$$

is injective.

Consider the diagram

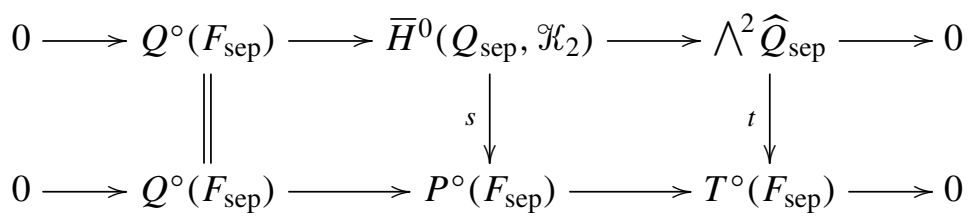

where $s$ is the composition of the natural map $\bar{H}^{0}\left(Q_{\text {sep }}, \mathscr{K}_{2}\right) \rightarrow \bar{H}^{0}\left(P_{\text {sep }}, \mathscr{K}_{2}\right)$ and a splitting of $P^{\circ}\left(F_{\text {sep }}\right) \rightarrow \bar{H}^{0}\left(P_{\text {sep }}, \mathscr{K}_{2}\right)$ (see Lemma 4.5).

We have the following diagram

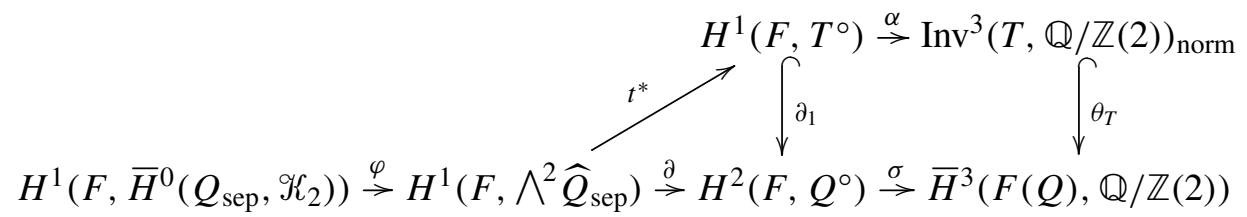

with the bottom sequence a complex, where $\sigma$ is the composition of the maps in (4-13) and (4-14):

$$
\begin{aligned}
H^{2}\left(F, Q^{0}\right) \stackrel{\psi}{\rightarrow} H^{2}\left(F, \bar{H}^{0}\left(Q_{\text {sep }}, \mathscr{K}_{2}\right)\right) & \stackrel{\beta}{\rightarrow} \bar{H}^{4}(Q, \mathbb{Z}(2)) \\
& \stackrel{\kappa}{\rightarrow} \bar{H}^{4}(F(Q), \mathbb{Z}(2))=\bar{H}^{3}(F(Q), \mathbb{Q} / \mathbb{Z}(2)),
\end{aligned}
$$

with $\varphi$ and $\psi$ given by Galois cohomology applied to the exact sequence (4-11) for the torus $Q$. Note that the connecting map $\partial_{1}$ is injective as $H^{1}\left(F, P^{\circ}\right)=0$ since $P^{\circ}$ is a quasisplit torus. As $2 \partial=0$ in (4-12), we have $2 t^{*}=0$.

The commutativity of the triangle follows from the definition of $t^{*}$. We claim that the square in the diagram is anticommutative. Note that $\partial_{2}(\xi)=\left[P_{\text {gen }}\right]$, where $\partial_{2}: H^{0}(F, Q) \rightarrow H^{1}(F, T)$ is the connecting homomorphism, $P_{\text {gen }}$ is the generic fiber of the morphism $P \rightarrow Q$, and $\xi \in H^{0}(K, Q)$ is the generic point of $Q$ with $K=F(Q)$. It follows from the description of the maps $\alpha$ and $\beta$ in (4-7) and (4-13), respectively, and Lemma A.1 that

$$
\sigma\left(\partial_{1}(a)\right)=\partial_{1}(a)_{K} \cup \xi=\left(-a_{K}\right) \cup \partial_{2}(\xi)=\left(-a_{K}\right) \cup\left[P_{\text {gen }}\right]=-\theta_{T}(\alpha(a))
$$

for every $a \in H^{1}\left(F, T^{\circ}\right)$.

The maps $\beta$ and $\kappa$ are injective, hence the bottom sequence in the diagram is 
exact. Thus, we have an exact sequence

$$
H^{1}\left(F, \bar{H}^{0}\left(Q_{\text {sep }}, \mathscr{K}_{2}\right)\right) \rightarrow H^{1}\left(F, \wedge^{2} \widehat{Q}_{\text {sep }}\right) \rightarrow \operatorname{Ker}(\alpha) \rightarrow 0
$$

and $2 \cdot \operatorname{Ker}(\alpha)=2 \cdot \operatorname{Im}\left(t^{*}\right)=0$. Furthermore, $\operatorname{Ker}(\alpha) \simeq \mathrm{CH}^{2}(B T)$ tors by Theorem 4.3 and the group $H^{1}\left(F, \bigwedge^{2} \widehat{Q}_{\text {sep }}\right)$ is finite as $\wedge^{2} \widehat{Q}_{\text {sep }}$ is a lattice.

We have proved:

Theorem 4.7. Let $1 \rightarrow T \rightarrow P \rightarrow Q \rightarrow 1$ be a coflasque resolution of a torus $T$. Then there is an exact sequence

$$
H^{1}\left(F, \bar{H}^{0}\left(Q_{\text {sep }}, \mathscr{K}_{2}\right)\right) \rightarrow H^{1}\left(F, \bigwedge^{2} \widehat{Q}_{\text {sep }}\right) \rightarrow \mathrm{CH}^{2}(B T)_{\text {tors }} \rightarrow 0 .
$$

Moreover, $\mathrm{CH}^{2}(B T)_{\text {tors }}$ is a finite group satisfying $2 \cdot \mathrm{CH}^{2}(B T)_{\mathrm{tors}}=0$.

Corollary 4.8. If $T^{\circ}$ is a birational direct factor of a rational torus, or if $T$ is split over a cyclic field extension, then $\mathrm{CH}^{2}(B T)_{\text {tors }}=0$, that is, the map $\alpha$ in Theorem 4.3 is injective.

Proof. The exact sequence $1 \rightarrow Q^{\circ} \rightarrow P^{\circ} \rightarrow T^{\circ} \rightarrow 1$ is a flasque resolution of $T^{\circ}$. If $T^{\circ}$ is a birational direct factor of a rational torus, or if $T$ is split over a cyclic field extension, the torus $Q^{\circ}$, and hence $Q$, is invertible; see Section 4a and [Voskresenskiur 1998, §4, Theorem 3]. By Lemma 4.5, the sequence (4-11) for the torus $Q$ is split, hence the first map in Theorem 4.7 is surjective.

Question 4.9. Is $\mathrm{CH}^{2}(B T)_{\text {tors }}$ trivial for every torus $T$ ?

4e. Special tori. Let $T$ be an algebraic torus over a field $F$. The tautological invariant of the torus $T^{\circ} \times T$ is the normalized invariant taking a pair

$$
(a, b) \in H^{1}\left(K, T^{\circ}\right) \times H^{1}(K, T)
$$

to the cup-product $a \cup b \in H^{3}(K, \mathbb{Q} / \mathbb{Z}(2))$ defined in (4-5).

The following theorem shows that if a torus $T$ has only trivial degree 3 normalized invariants with values in $\mathbb{Q} / \mathbb{Z}(2)$ universally, that is, over all field extensions of $F$, then $T$ has no nonconstant invariants at all by the simple reason: every $T$ torsor over a field is trivial. Note that it follows from Theorem 2.4 that $T$ has no degree 2 normalized invariants with values in $\mathbb{Q} / \mathbb{Z}(1)$ universally if and only if $T$ is coflasque.

Theorem 4.10. Let $T$ be an algebraic torus over a field $F$. Then the following are equivalent:

(1) $\operatorname{Inv}^{3}\left(T_{K}, \mathbb{Q} / \mathbb{Z}(2)\right)_{\text {norm }}=0$ for every field extension $K$ of $F$.

(2) The tautological invariant of the torus $T^{\circ} \times T$ is trivial.

(3) The torus $T$ is invertible.

(4) The torus $T$ is special. 
Proof. (1) $\Rightarrow$ (2): Let $K / F$ be a field extension and $a \in H^{1}\left(K, T^{\circ}\right)$. By assumption, the degree 3 normalized invariant $i=\alpha(a)$ with values in $\mathbb{Q} / \mathbb{Z}(2)$, defined by $i(b)=a \cup b$ for every $b \in H^{1}(K, T)$, is trivial. In other words, the tautological invariant of the torus $T^{\circ} \times T$ is trivial.

$(2) \Rightarrow(3)$ : The image of the tautological invariant in the group

$$
S^{2}\left(\widehat{T}_{\text {sep }}^{\circ} \oplus \widehat{T}_{\text {sep }}\right)^{\Gamma} / \text { Dec }
$$

is represented by the identity $1_{\widehat{T}}$ in the direct factor $\left(\widehat{T}_{\text {sep }}^{\circ} \otimes \widehat{T}_{\text {sep }}\right)^{\Gamma}=\operatorname{End}_{\Gamma}\left(\widehat{T}_{\text {sep }}\right)$ of $S^{2}\left(\widehat{T}_{\text {sep }}^{\circ} \oplus \widehat{T}_{\text {sep }}\right)^{\Gamma}$ (see Section A-II). The projection of Dec on the direct summand $\left(\widehat{T}_{\text {sep }}^{\circ} \otimes \widehat{T}_{\text {sep }}\right)^{\Gamma}$ is generated by the traces $\operatorname{Tr}(a \otimes b)$ for all open subgroups $\Gamma^{\prime} \subset \Gamma$ and all $a \in\left(\widehat{T}_{\mathrm{sep}}^{\circ}\right)^{\Gamma^{\prime}}$ and $b \in\left(\widehat{T}_{\mathrm{sep}}\right)^{\Gamma^{\prime}}$. Hence $1_{\widehat{T}}=\sum_{i} \operatorname{Tr}\left(a_{i} \otimes b_{i}\right)$ for some open subgroups $\Gamma_{i} \subset \Gamma, a_{i} \in\left(\widehat{T}^{\circ}\right)^{\Gamma_{i}}$ and $b_{i} \in(\widehat{T})^{\Gamma_{i}}$. If $P_{i}=\mathbb{Z}\left[\Gamma / \Gamma_{i}\right]$, then $a_{i}$ can be viewed as a $\Gamma$-homomorphism $\widehat{T} \rightarrow P_{i}$ and $b_{i}$ can be viewed as a $\Gamma$-homomorphism $P_{i} \rightarrow \widehat{T}$ such that the composition

$$
\widehat{T} \stackrel{\left(b_{i}\right)}{\longrightarrow} P \stackrel{\left(a_{i}\right)}{\longrightarrow} \widehat{T},
$$

where $P=\bigsqcup P_{i}$, is the identity. It follows that $\widehat{T}$ is a direct summand of a permutation $\Gamma$-module $P$ and hence $T$ is invertible.

(3) $\Rightarrow(4)$ : Obvious as every invertible torus is special.

(4) $\Rightarrow(1)$ : Obvious.

Remark 4.11. The equivalence (3) $\Leftrightarrow$ (4) was essentially proved in [ColliotThélène and Sansuc 1987b, Proposition 7.4].

4f. Linear and quadratic invariants. Let $T$ be a torus over $F$. By Theorem 4.3, we have a natural homomorphism to the group of linear invariants:

$$
\alpha: H^{1}\left(F, T^{\circ}\right) \rightarrow \operatorname{Inv}^{3}(T, \mathbb{Q} / \mathbb{Z}(2))_{\text {lin }} .
$$

Let $S$ and $T$ be algebraic tori over $F$. For every field extension $K / F$, the cup-product (4-2) yields a homomorphism

$$
\varepsilon:\left(\widehat{T}_{\mathrm{sep}}^{\otimes 2}\right)^{\Gamma} \rightarrow \operatorname{Inv}^{3}(T, \mathbb{Q} / \mathbb{Z}(2))
$$

defined by $\varepsilon(a)(b)=a_{K} \cup b \cup b$ for $a \in\left(\widehat{T}_{\mathrm{sep}}^{\otimes 2}\right)^{\Gamma}$ and $b \in H^{1}(K, T)$.

We say that an invariant $i \in \operatorname{Inv}^{3}(T, \mathbb{Q} / \mathbb{Z}(2))$ is quadratic if the function

$$
h(a, b):=i(a+b)-i(a)-i(b)
$$

is bilinear and $h(a, a)=2 i(a)$ for all $a$ and $b$. For example, the tautological invariant of the torus $T^{\circ} \times T$ in Section $4 \mathrm{e}$ is quadratic. We write $\operatorname{Inv}^{3}(T, \mathbb{Q} / \mathbb{Z}(2))_{\text {quad }}$ for the subgroup of all quadratic invariants in $\operatorname{Inv}^{3}(T, \mathbb{Q} / \mathbb{Z}(2))$. The image of $\varepsilon$ is contained in $\operatorname{Inv}^{3}(T, \mathbb{Q} / \mathbb{Z}(2))_{\text {quad }}$. 
Lemma 4.12. The composition of $\varepsilon$ with the map

$$
\operatorname{Inv}^{3}(T, \mathbb{Q} / \mathbb{Z}(2)) \rightarrow S^{2}\left(\widehat{T}_{\mathrm{sep}}\right)^{\Gamma} / \mathrm{Dec}
$$

in Theorem 4.3 is induced by the natural homomorphism $\widehat{T}_{\mathrm{sep}}^{\otimes 2} \rightarrow S^{2}\left(\widehat{T}_{\mathrm{sep}}\right)$.

Proof. Let $U \rightarrow U / T=: X$ be a standard classifying $T$-torsor as in Section 4c. Consider the commutative diagram

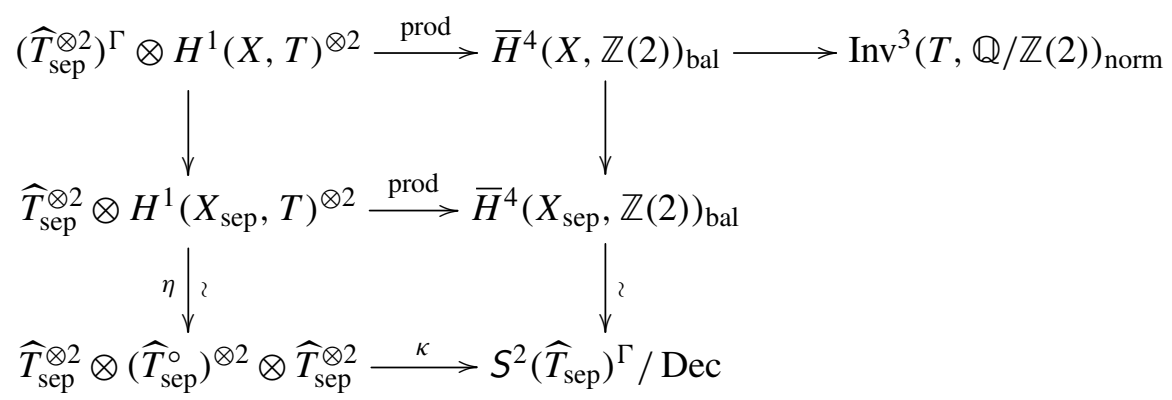

where the product maps are given by (4-1), $\eta$ identifies

$$
H^{1}\left(X_{\text {sep }}, T\right)=\widehat{T}_{\text {sep }}^{\circ} \otimes \operatorname{Pic}\left(X_{\text {sep }}\right)
$$

with $\widehat{T}_{\text {sep }}^{\circ} \otimes \widehat{T}_{\text {sep }}$ and $\kappa$ is given by the pairing between the first and second factors. Write $[U]$ for the class of the classifying torsor in $H^{1}(X, T)$. The image of $[U]$ in

$$
H^{1}\left(X_{\text {sep }}, T_{\text {sep }}\right)=\widehat{T}_{\text {sep }}^{\circ} \otimes \widehat{T}_{\text {sep }}=\operatorname{End}\left(\widehat{T}_{\text {sep }}\right)
$$

is the identity $1_{\widehat{T}_{\text {sep }}}$. Hence for every $a \in\left(\widehat{T}_{\text {sep }}^{\otimes 2}\right)^{\Gamma}$, the image of $a \otimes[U] \otimes[U]$ under the diagonal map in the diagram coincides with the canonical image of $a$ in $S^{2}\left(\widehat{T}_{\text {sep }}\right)^{\Gamma} /$ Dec.

The composition of the map $S^{2}\left(\widehat{T}_{\mathrm{sep}}\right)^{\Gamma} \rightarrow\left(\widehat{T}_{\mathrm{sep}}^{\otimes 2}\right)^{\Gamma}$ given by $a \cdot b \mapsto a \otimes b+$ $b \otimes a$ with the natural map $\left(\widehat{T}_{\text {sep }}^{\otimes 2}\right)^{\Gamma} \rightarrow S^{2}\left(\widehat{T}_{\text {sep }}\right)^{\Gamma}$ is multiplication by 2 . Then by Lemma 4.12, 2. $S^{2}\left(\widehat{T}_{\mathrm{sep}}\right)^{\Gamma} /$ Dec is contained in the image of the map

$$
\operatorname{Inv}^{3}(T, \mathbb{Q} / \mathbb{Z}(2)) \rightarrow S^{2}\left(\widehat{T}_{\text {sep }}\right)^{\Gamma} / \text { Dec }
$$

Theorem 4.3 then yields:

Theorem 4.13. Let $T$ be an algebraic torus over $F$. Then 2 times the homomorphism $S^{2}\left(\widehat{T}_{\mathrm{sep}}\right)^{\Gamma} / \operatorname{Dec} \rightarrow H^{2}\left(F, T^{0}\right)$ from Theorem 4.3 is trivial. If $p$ is an odd prime,

$$
\operatorname{Inv}^{3}\left(T, \mathbb{Q}_{p} / \mathbb{Z}_{p}(2)\right)_{\text {norm }}=\operatorname{Inv}^{3}\left(T, \mathbb{Q}_{p} / \mathbb{Z}_{p}(2)\right)_{\text {lin }} \oplus \operatorname{Inv}^{3}\left(T, \mathbb{Q}_{p} / \mathbb{Z}_{p}(2)\right)_{\text {quad }}
$$

and there are natural isomorphisms $\operatorname{Inv}^{3}\left(T, \mathbb{Q}_{p} / \mathbb{Z}_{p}(2)\right)_{\text {lin }} \simeq H^{1}\left(F, T^{\circ}\right)\{p\}$ and

$$
\operatorname{Inv}^{3}\left(T, \mathbb{Q}_{p} / \mathbb{Z}_{p}(2)\right)_{\text {quad }} \simeq\left(S^{2}\left(\widehat{T}_{\text {sep }}\right)^{\Gamma} / \text { Dec }\right)\{p\} .
$$


Example 4.14. Let $X=\left\{x_{1}, x_{2}, \ldots, x_{n}\right\}$ be a set of $n$ elements with the natural action of the symmetric group $S_{n}$. A continuous surjective group homomorphism $\Gamma \rightarrow S_{n}$ yields a separable field extension $L / F$ of degree $n$. Consider the torus $T=R_{L / F}\left(\mathbb{G}_{m, L}\right) / \mathbb{G}_{m}$, where $R_{L / F}$ is the Weil restriction; see [Voskresenskiu 1998, Chapter 1, §3.12]. Note that the generic maximal torus of the group $\mathbf{P G} \mathbf{L}_{n}$ is of this form (see Section $5 \mathrm{~b}$ ). The character lattice $\widehat{T}_{\text {sep }}$ is the kernel of the augmentation homomorphism $\mathbb{Z}[X] \rightarrow \mathbb{Z}$.

The dual torus $T^{\circ}$ is the norm one torus $R_{L / F}^{(1)}\left(\mathbb{G}_{m, L}\right)$. For every field extension $K / F$, we have:

$$
H^{1}(K, T)=\operatorname{Br}(K L / K), \quad H^{1}\left(K, T^{\circ}\right)=K^{\times} / N(K L)^{\times},
$$

where $K L:=K \otimes L, N$ is the norm map for the extension $K L / K$ and

$$
\operatorname{Br}(K L / K)=\operatorname{Ker}(\operatorname{Br}(K) \rightarrow \operatorname{Br}(K L)) .
$$

The pairing

$$
K^{\times} / N(K L)^{\times} \otimes \operatorname{Br}(K L / K) \rightarrow H^{3}(F, \mathbb{Q} / \mathbb{Z}(2))
$$

defines linear degree 3 invariants of both $T$ and $T^{\circ}$.

We claim that $S^{2}\left(\widehat{T}_{\text {sep }}\right)^{\Gamma} /$ Dec $=0$ and $S^{2}\left(\widehat{T}_{\text {sep }}^{\circ}\right)^{\Gamma} /$ Dec $=0$, that is, $T$ and $T^{\circ}$ have no nontrivial quadratic degree 3 invariants. We have $\widehat{T}_{\text {sep }}^{\circ}=\mathbb{Z}[X] / \mathbb{Z} N_{X}$, where $N_{X}=\sum x_{i}$. The group $S^{2}\left(\widehat{T}_{\text {sep }}^{\circ}\right)^{\Gamma}$ is generated by $S:=\sum_{i<j} x_{i} \cdot x_{j}$. As $S \in$ Dec, we have $S^{2}\left(\widehat{T}_{\text {sep }}^{\circ}\right)^{\Gamma} /$ Dec $=0$.

Let $D=\sum x_{i}^{2}$ and $E:=\operatorname{Qtr}\left(x_{1}-x_{2}\right)=2 S-(n-1) D$, where the quadratic map Qtr is defined in Section A-II. The group $S^{2}\left(\widehat{T}_{\mathrm{sep}}\right)^{\Gamma}$ is generated by $E$ if $n$ is even and by $E / 2$ if $n$ is odd. A computation shows that $n E / 2=\operatorname{Qtr}\left(n x_{1}-N_{X}\right)$. It follows that the generator of $S^{2}\left(\widehat{T}_{\text {sep }}\right)^{\Gamma}$ belongs to Dec, hence $S^{2}\left(\widehat{T}_{\text {sep }}\right)^{\Gamma} /$ Dec is trivial.

Note that as the torus $T$ is rational, it follows from Theorem 4.3 and Corollary 4.8 that $\operatorname{Inv}^{3}\left(T^{\circ}, \mathbb{Q} / \mathbb{Z}(2)\right)_{\text {norm }} \simeq \operatorname{Br}(L / F)$.

\section{Unramified invariants}

Let $K / F$ be a field extension and $v$ a discrete valuation of $K$ over $F$ with valuation ring $O_{v}$. We say that an element $a \in H^{n}(K, \mathbb{Q} / \mathbb{Z}(j))$ is unramified with respect to $v$ if $a$ belongs to the image of the map $H^{n}\left(O_{v}, \mathbb{Q} / \mathbb{Z}(j)\right) \rightarrow H^{n}(K, \mathbb{Q} / \mathbb{Z}(j))$; see [Colliot-Thélène and Ojanguren 1989]. We write $H_{\mathrm{nr}}^{n}(K, \mathbb{Q} / \mathbb{Z}(j))$ for the subgroup of the elements in $H^{n}(K, \mathbb{Q} / \mathbb{Z}(j))$ that are unramified with respect to all discrete valuations of $K$ over $F$. We have a natural homomorphism

$$
H^{n}(F, \mathbb{Q} / \mathbb{Z}(j)) \rightarrow H_{\mathrm{nr}}^{n}(K, \mathbb{Q} / \mathbb{Z}(j)) .
$$


A dominant morphism of varieties $Y \rightarrow X$ yields a homomorphism

$$
H_{\mathrm{nr}}^{n}(F(X), \mathbb{Q} / \mathbb{Z}(j)) \rightarrow H_{\mathrm{nr}}^{n}(F(Y), \mathbb{Q} / \mathbb{Z}(j)) .
$$

Proposition 5.1. Let $K / F$ be a purely transcendental field extension. Then the homomorphism (5-1) is an isomorphism.

Proof. The statement is well known for the $p$-components if $p \neq \operatorname{char} F$; see, for example, [Colliot-Thélène and Ojanguren 1989, Proposition 1.2]. It suffices to consider the case $K=F(t)$ and prove the surjectivity of (5-1). The coniveau spectral sequence for the projective line $\mathbb{P}^{1}$ (see (A-1) in the Appendix) yields an exact sequence

$$
H^{n}\left(\mathbb{P}^{1}, \mathbb{Q} / \mathbb{Z}(j)\right) \rightarrow H^{n}(K, \mathbb{Q} / \mathbb{Z}(j)) \rightarrow \coprod_{x \in \mathbb{P}^{1}} H_{x}^{n+1}\left(\mathbb{P}^{1}, \mathbb{Q} / \mathbb{Z}(j)\right)
$$

and, therefore, a surjective homomorphism $H^{n}\left(\mathbb{P}^{1}, \mathbb{Q} / \mathbb{Z}(j)\right) \rightarrow H_{\mathrm{nr}}^{n}(K, \mathbb{Q} / \mathbb{Z}(j))$. By the projective bundle theorem (classical if $p \neq \operatorname{char}(F)$ and [Gros 1985, Theorem 2.1.11] if $p=\operatorname{char}(F)>0$ ), we have

$$
H^{n}\left(\mathbb{P}^{1}, \mathbb{Q} / \mathbb{Z}(j)\right)=H^{n}(F, \mathbb{Q} / \mathbb{Z}(j)) \oplus H^{n-2}(F, \mathbb{Q} / \mathbb{Z}(j-1)) t,
$$

where $t$ is a generator of $H^{2}\left(\mathbb{P}^{1}, \mathbb{Z}(1)\right)=\operatorname{Pic}\left(\mathbb{P}^{1}\right)=\mathbb{Z}$. As $t$ vanishes over the generic point of $\mathbb{P}^{1}$, the result follows.

Let $G$ be a linear algebraic group over $F$. Choose a standard classifying $G$-torsor $U \rightarrow U / G$. An invariant $i \in \operatorname{Inv}^{n}(G, \mathbb{Q} / \mathbb{Z}(j))$ is called unramified if the image of $i$ under $\theta_{G}: \operatorname{Inv}^{n}(G, \mathbb{Q} / \mathbb{Z}(j)) \rightarrow H^{n}(F(U / G), \mathbb{Q} / \mathbb{Z}(j))$ is unramified. This is independent of the choice of standard classifying torsor. Indeed, if $U^{\prime} \rightarrow U^{\prime} / G$ is another standard classifying $G$-torsor, then $\left(U \times V^{\prime}\right) / G \rightarrow U / G$ and $\left(V \times U^{\prime}\right) / G \rightarrow U^{\prime} / G$ are vector bundles. Hence the field $F\left(\left(U \times U^{\prime}\right) / G\right)$ is a purely transcendental extension of $F(U / G)$ and $F\left(U^{\prime} / G\right)$ and by Proposition 5.1,

$$
\begin{aligned}
H_{\mathrm{nr}}^{n}(F(U / G), \mathbb{Q} / \mathbb{Z}(j)) & \simeq H_{\mathrm{nr}}^{n}\left(F\left(\left(U \times U^{\prime}\right) / G\right), \mathbb{Q} / \mathbb{Z}(j)\right) \\
& \simeq H_{\mathrm{nr}}^{n}\left(F\left(U^{\prime} / G\right), \mathbb{Q} / \mathbb{Z}(j)\right) .
\end{aligned}
$$

We write $H_{\mathrm{nr}}^{n}(F(B G), \mathbb{Q} / \mathbb{Z}(j))$ for this common value and $\operatorname{Inv}_{\mathrm{nr}}^{n}(G, \mathbb{Q} / \mathbb{Z}(j))$ for the subgroup of unramified invariants. Similarly, we write $\operatorname{Br}_{\mathrm{nr}}(F(B G))$ for the unramified Brauer group $H_{\mathrm{nr}}^{2}(F(B G), \mathbb{Q} / \mathbb{Z}(1))$.

Proposition 5.2. If $G^{\prime}$ be a subgroup of $G$ and $i \in \operatorname{Inv}_{\mathrm{nr}}^{n}(G, \mathbb{Q} / \mathbb{Z}(j))$, then

$$
\operatorname{res}(i) \in \operatorname{Inv}_{\mathrm{nr}}^{n}\left(G^{\prime}, \mathbb{Q} / \mathbb{Z}(j)\right) \text {. }
$$


Proof. It is shown in Section 2b that there is a surjective morphism $X^{\prime} \rightarrow X$ of the respective classifying varieties of $G^{\prime}$ and $G$, such that $\theta_{G}(i)_{F\left(X^{\prime}\right)}=\theta_{G^{\prime}}(\operatorname{res}(i))$. Applying the homomorphism (5-2) we see that $\operatorname{res}(i)$ is unramified.

Proposition 5.3. Let $G$ be a smooth linear algebraic group over a field $F$. The map $\operatorname{Inv}_{\mathrm{nr}}^{n}(G, \mathbb{Q} / \mathbb{Z}(j)) \rightarrow H_{\mathrm{nr}}^{n}(F(B G), \mathbb{Q} / \mathbb{Z}(j))$ induced by $\theta_{G}$ is an isomorphism.

Proof. By Theorem 3.4, it suffices to show that

$$
H_{\mathrm{nr}}^{n}(F(U / G), \mathbb{Q} / \mathbb{Z}(j)) \subset H^{n}(F(U / G), \mathbb{Q} / \mathbb{Z}(j))_{\mathrm{bal}} .
$$

We follow Totaro's approach; see [Garibaldi et al. 2003, p. 99]. Consider the open subscheme $W$ of $\left(U^{2} / G\right) \times \mathbb{A}^{1}$ of all triples $\left(u, u^{\prime}, t\right)$ such that $(2-t) u+(t-1) u^{\prime} \in U$. We have the projection $q: W \rightarrow U^{2} / G$, the morphisms $f: W \rightarrow U / G$ defined by $f\left(u, u^{\prime}, t\right)=(2-t) u+(t-1) u^{\prime}$, and $h_{i}: U^{2} / G \rightarrow W$ defined by $h_{i}\left(u, u^{\prime}\right)=\left(u, u^{\prime}, i\right)$ for $i=1$ and 2. The composition $f \circ h_{i}$ is the projection $p_{i}: U^{2} / G \rightarrow U / G$ and $q \circ h_{i}$ is the identity of $U^{2} / G$.

Let $w_{i}$ be the generic point of the preimage of $i$ with respect to the projection $W \rightarrow \mathbb{A}^{1}$ and write $O_{i}$ for the local ring of $W$ at $w_{i}$. The morphisms $q, f$, and $h_{i}$ yield $F$-algebra homomorphisms $F\left(U^{2} / G\right) \rightarrow O_{i}, F(U / G) \rightarrow O_{i}$ and $O_{i} \rightarrow F(U / G)$. Note that by Proposition A.11, we have

$$
H_{\mathrm{nr}}^{n}(F(W), \mathbb{Q} / \mathbb{Z}(j)) \subset H^{n}\left(O_{i}, \mathbb{Q} / \mathbb{Z}(j)\right) .
$$

In the commutative diagram

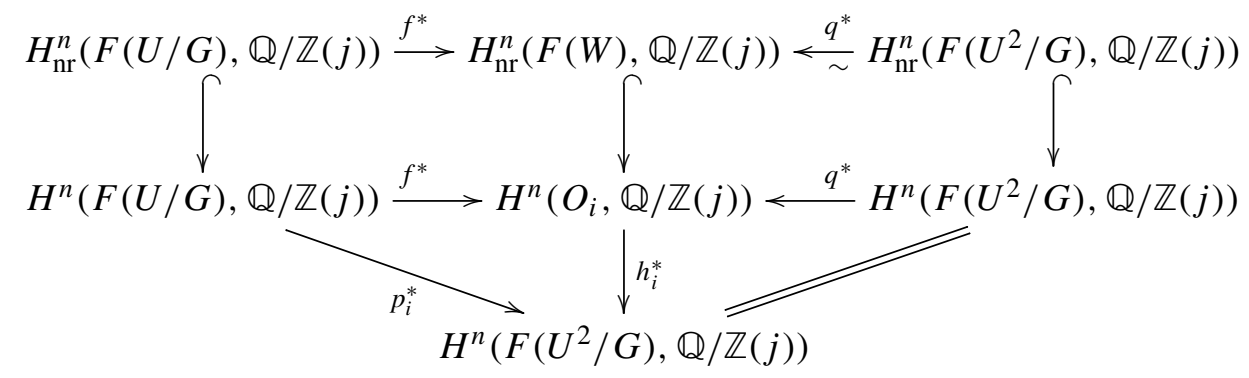

the top right map $q^{*}$ is an isomorphism by Proposition 5.1 since the field extension $F(W) / F\left(U^{2} / G\right)$ is purely transcendental. It follows that the restriction of $p_{i}^{*}$ on $H_{\mathrm{nr}}^{n}(F(U / G), \mathbb{Q} / \mathbb{Z}(j))$ coincides with $\left(q^{*}\right)^{-1} \circ f^{*}$ and hence is independent of $i$.

\section{5a. Unramified invariants of tori.}

Proposition 5.4. If $T$ is a flasque torus, then every invariant in $\operatorname{Inv}^{n}(T, \mathbb{Q} / \mathbb{Z}(j))$ is unramified. 
Proof. Consider an exact sequence of tori $1 \rightarrow T \rightarrow P \rightarrow Q \rightarrow 1$ with $P$ quasisplit. Choose a smooth projective compactification $X$ of $Q$; see [Colliot-Thélène et al. 2005]. As $T$ is flasque, by [Colliot-Thélène and Sansuc 1977, Proposition 9], there is a $T$-torsor $E \rightarrow X$ extending the $T$-torsor $P \rightarrow Q$. The torsor $E$ is classifying and $T$-rational. Choose an invariant in $\operatorname{Inv}^{n}(G, \mathbb{Q} / \mathbb{Z}(j))$ and consider its image $a$ in $H^{n}(F(X), \mathbb{Q} / \mathbb{Z}(j))_{\text {bal }}$ (see Theorem 3.4). We show that $a$ is unramified with respect to every discrete valuation $v$ on $F(X)$ over $F$; see [Colliot-Thélène 1995, Proposition 2.1.8]. By Proposition A.9, $a$ is unramified with respect to the discrete valuation associated to every point $x \in X$ of codimension 1 , that is, $\partial_{x}(a)=0$.

As $X$ is projective, the valuation ring $O_{v}$ of the valuation $v$ dominates a point $x \in X$. It follows from Proposition A.11 that $a$ belongs to the image of

$$
H^{n}\left(O_{X, x}, \mathbb{Q} / \mathbb{Z}(j)\right) \rightarrow H^{n}(F(X), \mathbb{Q} / \mathbb{Z}(j)) .
$$

As the local ring $O_{X, x}$ is a subring of $O_{v}, a$ belongs to the image of

$$
H^{n}\left(O_{v}, \mathbb{Q} / \mathbb{Z}(j)\right) \rightarrow H^{n}(F(X), \mathbb{Q} / \mathbb{Z}(j))
$$

and hence $a$ is unramified with respect to $v$.

Let $T$ be a torus over $F$. By [Colliot-Thélène and Sansuc 1987b, Lemma 0.6], there is an exact sequence of tori $1 \rightarrow T \rightarrow T^{\prime} \rightarrow P \rightarrow 1$ with $T^{\prime}$ flasque and $P$ quasisplit. The following theorem computes the unramified invariants of $T$ in terms of the invariants of $T^{\prime}$.

Theorem 5.5. There is a natural isomorphism

$$
\operatorname{Inv}_{\mathrm{nr}}^{n}(T, \mathbb{Q} / \mathbb{Z}(j)) \simeq \operatorname{Inv}^{n}\left(T^{\prime}, \mathbb{Q} / \mathbb{Z}(j)\right) .
$$

Proof. Choose an exact sequence $1 \rightarrow T^{\prime} \rightarrow P^{\prime} \rightarrow S \rightarrow 1$ with $P^{\prime}$ a quasisplit torus. Let $S^{\prime}$ be the cokernel of the composition $T \rightarrow T^{\prime} \rightarrow P^{\prime}$. We have an exact sequence $1 \rightarrow P \rightarrow S^{\prime} \rightarrow S \rightarrow 1$. As $P$ is quasisplit, the latter exact sequence splits at the generic point of $S$ and therefore, $F\left(S^{\prime}\right)$ is a purely transcendental field extension of $F(S)$. It follows from Propositions 5.1, 5.3, and 5.4 that

$$
\begin{aligned}
\operatorname{Inv}_{\mathrm{nr}}^{n}(T, \mathbb{Q} / \mathbb{Z}(j)) & \simeq H_{\mathrm{nr}}^{n}\left(F\left(S^{\prime}\right), \mathbb{Q} / \mathbb{Z}(j)\right) \simeq H_{\mathrm{nr}}^{n}(F(S), \mathbb{Q} / \mathbb{Z}(j)) \\
& \simeq \operatorname{Inv}_{\mathrm{nr}}^{n}\left(T^{\prime}, \mathbb{Q} / \mathbb{Z}(j)\right)=\operatorname{Inv}^{n}\left(T^{\prime}, \mathbb{Q} / \mathbb{Z}(j)\right) .
\end{aligned}
$$

The following corollary is essentially equivalent to [Colliot-Thélène and Sansuc 1987b, Proposition 9.5] in the case when $F$ is of zero characteristic.

Corollary 5.6. With notation as above, the isomorphism

$$
\operatorname{Inv}(T, \operatorname{Br}) \stackrel{\sim}{\longrightarrow} \operatorname{Pic}(T)=H^{1}(F, \widehat{T})
$$


identifies $\operatorname{Inv}_{\mathrm{nr}}(T, \mathrm{Br})$ with the subgroup $H^{1}\left(F, \widehat{T}^{\prime}\right)$ of $H^{1}(F, \widehat{T})$ of all elements that are trivial when restricted to all cyclic subgroups of the decomposition group of $T$.

Proof. The description of $H^{1}\left(F, \widehat{T}^{\prime}\right)$ as a subgroup of $H^{1}(F, \widehat{T})$ is given in [ColliotThélène and Sansuc 1987b, Proposition 9.5], and this part of the proof is characteristic free.

In view of Propositions 5.1 and 5.3 we can calculate the group of unramified cohomology for the function field of an arbitrary torus in terms of the invariants of a flasque torus:

Theorem 5.7. Let $S$ be a torus over $F$ and let $1 \rightarrow T \rightarrow P \rightarrow S \rightarrow 1$ be a flasque resolution of $S$, that is, $T$ is flasque and $P$ is quasisplit. Then there is a natural isomorphism

$$
H_{\mathrm{nr}}^{n}(F(S), \mathbb{Q} / \mathbb{Z}(j)) \simeq \operatorname{Inv}^{n}(T, \mathbb{Q} / \mathbb{Z}(j)) .
$$

Note that the torus $S$ determines $T$ up to multiplication by a quasisplit torus. If $X$ is a smooth compactification of $S$, then one can take a torus $T$ with $\widehat{T}_{\mathrm{sep}} \simeq \operatorname{Pic}\left(X_{\mathrm{sep}}\right)$; see [Colliot-Thélène and Sansuc 1977, Proposition 6; Voskresenskiǔ 1998, §4.6].

Corollary 5.8. A torus $S$ has no nonconstant unramified degree 3 cohomology with values in $\mathbb{Q} / \mathbb{Z}(2)$ universally, that is, $H_{\mathrm{nr}}^{3}(K(S), \mathbb{Q} / \mathbb{Z}(2))=H^{3}(K, \mathbb{Q} / \mathbb{Z}(2))$ for any field extension $K / F$, if and only if $S$ is a direct factor of a rational torus.

Proof. If $S$ is a direct factor of a rational torus, then $S$ has no nonconstant unramified cohomology by Proposition 5.1.

Conversely, let $1 \rightarrow T \rightarrow P \rightarrow S \rightarrow 1$ be a flasque resolution of $S$. By Theorem 5.7, $\operatorname{Inv}^{3}\left(T_{K}, \mathbb{Q} / \mathbb{Z}(2)\right)_{\text {norm }}=0$ for every $K / F$. It follows from Theorem 4.10 that $T$ is invertible and hence $S$ is a factor of a rational torus (see Section 4a).

Theorems 4.3, 5.7 and [Colliot-Thélène and Sansuc 1977, §2] yield the following proposition.

Proposition 5.9. Let $S$ be a torus over $F$ and let $1 \rightarrow T \rightarrow P \rightarrow S \rightarrow 1$ be a flasque resolution of $S$. Then we have an exact sequence

$$
\begin{aligned}
0 \rightarrow \mathrm{CH}^{2}(B T)_{\text {tors }} \rightarrow H^{1}\left(F, T^{0}\right) \rightarrow \bar{H}_{\mathrm{nr}}^{3}(F(S), \mathbb{Q} / \mathbb{Z}(2)) \\
\rightarrow H^{0}\left(F, S^{2}\left(\widehat{T}_{\mathrm{sep}}\right)\right) / \operatorname{Dec} \rightarrow H^{2}\left(F, T^{0}\right) .
\end{aligned}
$$

For an odd prime p, there is a canonical direct sum decomposition

$$
\bar{H}_{\mathrm{nr}}^{3}\left(F(S), \mathbb{Q}_{p} / \mathbb{Z}_{p}(2)\right)=H^{1}\left(F, T^{0}\right)\{p\} \oplus\left(H^{0}\left(F, S^{2}\left(\widehat{T}_{\mathrm{sep}}\right)\right) / \operatorname{Dec}\right)\{p\} .
$$

If $X$ is a smooth compactification of $S$, one can take the torus $T$ with $\widehat{T}_{\mathrm{sep}}=\operatorname{Pic}\left(X_{\mathrm{sep}}\right)$. 
5b. The Brauer invariant for semisimple groups. The following theorem was proved by Bogomolov [1987, Lemma 5.7] in characteristic zero:

Theorem 5.10. Let $G$ be a (connected) semisimple group over a field $F$. Then $\operatorname{Inv}_{\mathrm{nr}}(G, \mathrm{Br})=\operatorname{Inv}(G, \mathrm{Br})_{\text {const }}=\operatorname{Br}(F)$ and $\operatorname{Br}_{\mathrm{nr}}(F(B G))=\operatorname{Br}(F)$.

Proof. Let $G^{\prime} \rightarrow G$ be a simply connected cover of $G$ and $C$ the kernel of $G^{\prime} \rightarrow G$. By Theorem 2.4 and [Sansuc 1981, Lemme 6.9(iii)], we have

$$
\operatorname{Inv}(G, \operatorname{Br})_{\text {norm }}=\operatorname{Pic}(G)=\widehat{C}(F) .
$$

As the map $\widehat{C}(F) \rightarrow \widehat{C}\left(F_{\text {sep }}\right)$ is injective, we can replace $F$ by $F_{\text {sep }}$ and assume that the group $G$ is split.

Consider the variety $\mathscr{T}$ of maximal tori in $G$ and the closed subscheme $\mathscr{X} \subset G \times \mathscr{T}$ of all pairs $(g, T)$ with $g \in T$. The generic fiber of the projection $\mathscr{X} \rightarrow \mathscr{T}$ is the generic torus $T_{\mathrm{gen}}$ of $G$. Then $T_{\mathrm{gen}}$ is a maximal torus of $G_{K}$, where $K:=F(\mathscr{T})$.

Every maximal torus in $G$ is the factor torus of a maximal torus in $G^{\prime}$ by $C$. It follows that the variety $\mathscr{T}^{\prime}$ of maximal tori in $G^{\prime}$ is naturally isomorphic to $\mathscr{T}$. Moreover, as the generic torus $T_{\mathrm{gen}}^{\prime}$ of $G^{\prime}$ is a maximal torus of $G_{K}^{\prime}$, we have $T_{\mathrm{gen}} \simeq T_{\mathrm{gen}}^{\prime} / C_{K}$ and, therefore, an exact sequence of character groups

$$
0 \rightarrow \widehat{T}_{\text {gen }} \rightarrow \widehat{T}_{\text {gen }}^{\prime} \rightarrow \widehat{C}_{K} \rightarrow 0 .
$$

By Theorem 2.4, the composition of the natural homomorphism

$$
\operatorname{Inv}(G, \mathrm{Br})_{\text {norm }} \rightarrow \operatorname{Inv}\left(G_{K}, \mathrm{Br}\right)_{\text {norm }}
$$

with the restriction $\operatorname{Inv}\left(G_{K}, \mathrm{Br}\right)_{\text {norm }} \rightarrow \operatorname{Inv}\left(T_{\text {gen }}, \mathrm{Br}\right)_{\text {norm }}$ can be identified with the natural composition $\operatorname{Pic}(G) \rightarrow \operatorname{Pic}\left(G_{K}\right) \rightarrow \operatorname{Pic}\left(T_{\text {gen }}\right)$ and hence with the connecting homomorphism $\widehat{C}(F)=\widehat{C}(K) \rightarrow H^{1}\left(K, \widehat{T}_{\text {gen }}\right)$. Note that as $F=F_{\text {sep }}$, the decomposition group of $T_{\text {gen }}$ coincides with the Weyl group $W$ of $G$ by [Voskresenskiı 1988, Theorem 1], hence $H^{1}\left(K, \widehat{T}_{\text {gen }}\right) \simeq H^{1}\left(W, \widehat{T}_{\text {gen }}\right)$.

Let $w$ be a Coxeter element in $W{ }^{1}{ }^{1}$ It is the product of reflections with respect to all simple roots (in some order). By [Humphreys 1990, Lemma, p. 76], 1 is not an eigenvalue of $w$ on the space of weights $\widehat{T}^{\prime}$ gen $\otimes \mathbb{R}$. Let $W_{0}$ be the cyclic subgroup in $W$ generated by $w$. It follows that the first term in the exact sequence

$$
\left(\widehat{T}^{\prime} \text { gen }\right)^{W_{0}} \rightarrow \widehat{C}(K) \rightarrow H^{1}\left(W_{0}, \widehat{T}_{\text {gen }}\right)
$$

is trivial, that is, the second map is injective. Hence every nonzero character $\chi$ in $\widehat{C}(K)$ restricts to a nonzero element in $H^{1}\left(W_{0}, \widehat{T}_{\text {gen }}\right)$. It follows that the image of $\chi$ in $H^{1}\left(W, \widehat{T}_{\text {gen }}\right)$ is ramified by Corollary 5.6, hence so is $\chi$ by Proposition 5.2.

\footnotetext{
${ }^{1}$ We owe the idea to use the Coxeter element and the reference below to S. Garibaldi.
} 


\section{Appendix A: Generalities}

A-I: Proof of Theorem 2.2. Suppose that $i\left(E_{\mathrm{gen}}\right)=0$ for an $H$-invariant $i$ of $G$. Let $K / F$ be a field extension and $I \rightarrow \operatorname{Spec} K$ a $G$-torsor. We need to show that $i(I)=0$ in $H(K)$.

Suppose first that $K$ is infinite. Find a point $x \in X(K)$ such that $I$ is isomorphic to the pull-back of the classifying torsor with respect to $x$. Let $x^{\prime}$ be a rational point of $X_{K}$ above $x$ and write $O$ for the local ring $O_{X_{K}, x^{\prime}}$. The $K$-algebra $O$ is a regular local ring with residue field $K$. Therefore, the completion $\widehat{O}$ is isomorphic to $K \llbracket t_{1}, t_{2}, \ldots, t_{n} \rrbracket$ over $K$. Let $L$ be the quotient field of $\widehat{O}$, a field extension of $K(X)$. We have the following diagram of morphisms with a commutative square and three triangles:

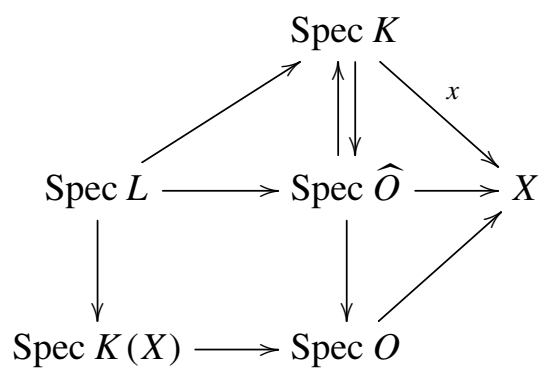

The pull-back of the classifying torsor $E \rightarrow X$ via $\operatorname{Spec} K(X) \rightarrow X$ is $\left(E_{\text {gen }}\right)_{K(X)}$. The $G$-torsor $I$ is the pull-back of $E \rightarrow X$ with respect to $X$. Let $\widehat{E}$ be the pull-back of $E \rightarrow X$ via Spec $\widehat{O} \rightarrow X$. Therefore, $I$ is the pull-back of $\widehat{E}$. Since $G$ is smooth, by a theorem of Grothendieck [Demazure and Grothendieck 1970, XXIV, Proposition 8.1], $\widehat{E}$ is the pull-back of $I$ with respect to $\operatorname{Spec} \widehat{O} \rightarrow \operatorname{Spec}(K)$. It follows that $I_{L} \simeq\left(E_{\mathrm{gen}}\right)_{L}$ as torsors over $L$. Hence the images of $i(I)$ and $i\left(E_{\text {gen }}\right)$ in $H(L)$ are equal and therefore, $i(I)_{L}=0$. By Property 2.1, we have $i(I)=0$.

If $K$ is finite, we replace $F$ by $F((t))$ and $K$ by $K((t))$. By the first part of the proof, $i(I)$ belongs to the kernel of $H(K) \rightarrow H(K((t)))$ and hence is trivial by Property 2.1 again.

A-II: Decomposable elements. Let $\Gamma$ be a profinite group and $A$ a $\Gamma$-lattice. Write $A^{\Gamma}$ for the subgroup of $\Gamma$-invariant elements in $A$. Let $\Gamma^{\prime} \subset \Gamma$ be an open subgroup and choose representatives $\gamma_{1}, \gamma_{2}, \ldots, \gamma_{n}$ for the left cosets of $\Gamma^{\prime}$ in $\Gamma$. We have the trace map $\operatorname{Tr}: A^{\Gamma^{\prime}} \rightarrow A^{\Gamma}$ defined by $\operatorname{Tr}(a)=\sum_{i=1}^{n} \gamma_{i} a$.

Let $S^{2}(A)$ be the symmetric square of $A$. Consider the quadratic trace map Qtr : $A^{\Gamma^{\prime}} \rightarrow S^{2}(A)^{\Gamma}$ defined by $\operatorname{Qtr}(a)=\sum_{i<j}\left(\gamma_{i} a\right)\left(\gamma_{j} a\right)$. Write $\operatorname{Dec}(A)$ for the subgroup of decomposable elements in $S^{2}(A)^{\Gamma}$ generated by the square $\left(A^{\Gamma}\right)^{2}$ of $A^{\Gamma}$ and the elements $\operatorname{Qtr}(a)$ for all open subgroups $\Gamma^{\prime} \subset \Gamma$ and all $a \in A^{\Gamma^{\prime}}$. 
Let $B$ be another $\Gamma$-lattice. We write $\operatorname{Dec}(A, B)$ for the subgroup of $(A \otimes B)^{\Gamma}$ generated by elements of the form $\operatorname{Tr}(a \otimes b)$ for all open subgroups $\Gamma^{\prime} \subset \Gamma$ and all $a \in A^{\Gamma^{\prime}}, b \in B^{\Gamma^{\prime}}$.

There is a natural isomorphism $S^{2}(A \oplus B) \simeq S^{2}(A) \oplus(A \otimes B) \oplus S^{2}(B)$. Moreover, the equality $\operatorname{Qtr}(a+b)=\mathrm{Q} \operatorname{tr}(a)+(\operatorname{Tr}(a) \otimes \operatorname{Tr}(b)-\operatorname{Tr}(a \otimes b))+\mathrm{Q} \operatorname{tr}(b)$ yields the decomposition

$$
\operatorname{Dec}(A \oplus B) \simeq \operatorname{Dec}(A) \oplus \operatorname{Dec}(A, B) \oplus \operatorname{Dec}(B) .
$$

A-III: Cup-products. Let $1 \rightarrow T \rightarrow P \rightarrow Q \rightarrow 1$ be an exact sequence of tori. We consider the connecting maps

$$
\partial_{1}: H^{p}(F, \widehat{T}(i)) \rightarrow H^{p+1}(F, \widehat{Q}(i))
$$

for the exact sequence $0 \rightarrow \widehat{Q}_{\text {sep }} \rightarrow \widehat{P}_{\text {sep }} \rightarrow \widehat{T}_{\text {sep }} \rightarrow 0$ of character $\Gamma$-lattices and

$$
\partial_{2}: H^{q}\left(F, \widehat{Q}^{\circ}(j)\right) \rightarrow H^{q+1}\left(F, \widehat{T}^{\circ}(j)\right)
$$

for the dual sequence of lattices (see notation in Section $4 b$ ).

Lemma A.1. Let $a \in H^{p}(F, \widehat{T}(i))$ and $b \in H^{q}\left(F, \widehat{Q}^{\circ}(j)\right)$ with $i+j \leq 2$. Then $\partial_{1}(a) \cup b=(-1)^{p+1} a \cup \partial_{2}(b)$ in $H^{p+q+1}(F, \mathbb{Z}(i+j))$, where the cup-product is defined in (4-3).

Proof. By [Cartan and Eilenberg 1999, Chapter V, Proposition 4.1], the elements $\partial_{1}\left(1_{T}\right)$ and $\partial_{2}\left(1_{Q}\right)$ in

$$
H^{1}\left(F, \widehat{T}_{\mathrm{sep}}^{\circ} \otimes \widehat{Q}_{\mathrm{sep}}\right)=\operatorname{Ext}_{\Gamma}^{1}\left(\widehat{T}_{\mathrm{sep}}, \widehat{Q}_{\mathrm{sep}}\right)
$$

differ by a sign. Write $\tau$ for the isomorphism induced by permutation of the factors. By the standard properties of the cup-product, we have

$$
\begin{aligned}
\partial_{1}(a) \cup b & =1_{T} \cup \partial_{1}(a) \cup b=\partial_{1}\left(1_{T}\right) \cup a \cup b=(-1)^{p q} \tau\left(\partial_{1}\left(1_{T}\right) \cup b \cup a\right) \\
& =(-1)^{p q+1} \tau\left(\partial_{2}\left(1_{Q}\right) \cup b \cup a\right)=(-1)^{p q+1} \tau\left(1_{Q} \cup \partial_{2}(b) \cup a\right) \\
& =(-1)^{p+1} 1_{Q} \cup a \cup \partial_{2}(b)=(-1)^{p+1} a \cup \partial_{2}(b) .
\end{aligned}
$$

A-IV: Cosimplicial abelian groups. Let $A^{\bullet}$ be a cosimplicial abelian group

$$
A^{0} \underset{d^{1}}{\stackrel{d^{0}}{\rightleftarrows}} A^{1} \Longrightarrow A^{2} \underset{\Longrightarrow}{\rightrightarrows}
$$

and write $h_{*}\left(A^{\bullet}\right)$ for the homology groups of the associated complex of abelian groups. In particular,

$$
h_{0}\left(A^{\bullet}\right)=\operatorname{Ker}\left[\left(d^{0}-d^{1}\right): A^{0} \rightarrow A^{1}\right] .
$$


We say that the cosimplicial abelian group $A^{\bullet}$ is constant if for every $i$, all the coface maps $d_{j}: A^{i-1} \rightarrow A^{i}, j=0,1, \ldots, i$, are isomorphisms. In this case all the $d_{j}$ are equal as $d_{j}=s_{j}^{-1}=d_{j+1}$, where the $s_{j}$ are the codegeneracy maps. For a constant cosimplicial abelian group $A^{\bullet}$, we have $h_{0}\left(A^{\bullet}\right)=A^{0}$ and $h_{i}\left(A^{\bullet}\right)=0$ for all $i>0$. We will need the following straightforward statement.

Lemma A.2. Let $0 \rightarrow A^{\bullet} \rightarrow B^{\bullet} \rightarrow C^{\bullet} \rightarrow D^{\bullet}$ be an exact sequence of cosimplicial abelian groups with $A^{\bullet}$ a constant cosimplicial group. Then the sequence of groups $0 \rightarrow A^{0} \rightarrow h_{0}\left(B^{\bullet}\right) \rightarrow h_{0}\left(C^{\bullet}\right) \rightarrow h_{0}\left(D^{\bullet}\right)$ is exact.

Let $H$ be a contravariant functor from the category of schemes over $F$ to the category of abelian groups. We say that $H$ is homotopy invariant if for every vector bundle $E \rightarrow X$ over $F$, the induced map $H(X) \rightarrow H(E)$ is an isomorphism.

For an integer $d>0$ consider the following property of the functor $H$ :

Property A.3. For every closed subscheme $Z$ of a scheme $X$ with $\operatorname{codim}_{X}(Z) \geq d$, the natural homomorphism $H(X) \rightarrow H(X \backslash Z)$ is an isomorphism.

Let $G$ be a linear algebraic group over a field $F$ and choose a standard classifying $G$-torsor $U \rightarrow U / G$. Let $U^{i}$ denote the product of $i$ copies of $U$. We have the $G$-torsors $U^{i} \rightarrow U^{i} / G$.

Consider the cosimplicial abelian group $A^{\bullet}=H\left(U^{\bullet} / G\right)$ with $A^{i}=H\left(U^{i+1} / G\right)$ and coface maps $A^{i-1} \rightarrow A^{i}$ induced by the projections $U^{i+1} / G \rightarrow U^{i} / G$.

Lemma A.4. Let $H$ be a homotopy invariant functor satisfying Property A.3 for some d. Let $U \rightarrow U / G$ be a standard classifying $G$-torsor and $U^{\prime}$ an open subset of a $G$-representation $V^{\prime}$.

1. If $\operatorname{codim}_{V^{\prime}}\left(V^{\prime} \backslash U^{\prime}\right) \geq d$, then the natural homomorphism

$$
H(U / G) \rightarrow H\left(\left(U \times U^{\prime}\right) / G\right)
$$

is an isomorphism.

2. If $\operatorname{codim}_{V}(V \backslash U) \geq d$, then the cosimplicial group $H\left(U^{\bullet} / G\right)$ is constant.

Proof. 1. The scheme $\left(U \times U^{\prime}\right) / G$ is an open subset of the vector bundle $\left(U \times V^{\prime}\right) / G$ over $U / G$ with complement of codimension at least $d$. The map in question is the composition $H(U / G) \rightarrow H\left(\left(U \times V^{\prime}\right) / G\right) \rightarrow H\left(\left(U \times U^{\prime}\right) / G\right)$ and both maps in the composition are isomorphisms since $H$ is homotopy invariant and satisfies Property A.3.

2. By the first part of the lemma applied to the $G$-torsor $U^{i} \rightarrow U^{i} / G$ and $U^{\prime}=U$, the map $H\left(U^{i} / G\right) \rightarrow H\left(U^{i+1} / G\right)$ induced by a projection $U^{i+1} / G \rightarrow U^{i} / G$ is an isomorphism.

By Lemma A.4, if $H$ is a homotopy invariant functor satisfying Property A.3 for some $d$, then the group $H(U / G)$ does not depend on the choice of the representation 
$V$ and the open set $U \subset V$ provided $\operatorname{codim}_{V}(V \backslash U) \geq d$. Following [Totaro 1999], we denote this group by $H(B G)$.

Example A.5. The split torus $T=\left(\mathbb{G}_{m}\right)^{n}$ over $F$ acts freely on the product $U$ of $n$ copies of $\mathbb{A}^{r+1} \backslash\{0\}$ with $U / T \simeq\left(\mathbb{P}^{r}\right)^{n}$, that is, $B T$ is "approximated" by the varieties $\left(\mathbb{P}^{r}\right)^{n}$ if " $r \gg 0$." We then have $\mathrm{CH}^{*}(B T)=S^{*}(\widehat{T})$, where $S^{*}$ represents the symmetric algebra and $\widehat{T}$ is the character group of $T$; see [Edidin and Graham 1998, p. 607]. In particular, $\operatorname{Pic}(B T)=\mathrm{CH}^{1}(B T)=\widehat{T}$. More generally, by the Künneth formula [Esnault et al. 1998, Proposition 3.7],

$$
H_{\mathrm{Zar}}^{*}\left(B T, \mathcal{K}_{*}\right) \simeq \mathrm{CH}^{*}(B T) \otimes K_{*}(F) \simeq S^{*}(\widehat{T}) \otimes K_{*}(F),
$$

where $K_{n}(F)$ is the Quillen $K$-group of $F$ and $\mathscr{K}_{n}$ is the Zariski sheaf associated to the presheaf $U \mapsto K_{n}(U)$.

A-V: Étale cohomology. For a scheme $X$ and a closed subscheme $Z \subset X$ we write $H_{Z}^{*}(X, \mathbb{Q} / \mathbb{Z}(j))$ for the étale cohomology group of $X$ with support in $Z$ and values in $\mathbb{Q} / \mathbb{Z}(j)$ [Milne 1980, Chapter III, §1]. Write $X^{(i)}$ for the set of points in $X$ of codimension $i$. For a point $x \in X^{(1)}$ set

$$
H_{x}^{*}(X, \mathbb{Q} / \mathbb{Z}(j))=\operatorname{colim}_{x \in U} H_{\{x\} \cap U}^{*}(U, \mathbb{Q} / \mathbb{Z}(j)),
$$

where the colimit is taken over all open subsets $U \subset X$ containing $x$. If $X$ is a variety, write

$$
\partial_{x}: H^{*}(F(X), \mathbb{Q} / \mathbb{Z}(j)) \rightarrow H_{x}^{*+1}(X, \mathbb{Q} / \mathbb{Z}(j))
$$

for the residue homomorphisms arising from the coniveau spectral sequence [ColliotThélène et al. 1997, 1.2]

$$
E_{1}^{p, q}=\coprod_{x \in X^{(p)}} H_{x}^{p+q}(X, \mathbb{Q} / \mathbb{Z}(j)) \Rightarrow H^{p+q}(X, \mathbb{Q} / \mathbb{Z}(j)) .
$$

Let $f: Y \rightarrow X$ be a dominant morphism of varieties over $F, y \in Y^{(1)}$, and $x=f(y)$. If $x \in X^{(1)}$, there is a natural homomorphism

$$
f_{y}^{*}: H_{x}^{*}(X, \mathbb{Q} / \mathbb{Z}(j)) \rightarrow H_{y}^{*}(Y, \mathbb{Q} / \mathbb{Z}(j)) .
$$

The following lemma is straightforward.

Lemma A.6. Let $f: Y \rightarrow X$ be a dominant morphism of varieties over $F, y \in Y^{(1)}$ and $x=f(y)$.

(1) If $x$ is the generic point of $X$, then the composition

$$
H^{*}(F(X), \mathbb{Q} / \mathbb{Z}(j)) \stackrel{f^{*}}{\rightarrow} H^{*}(F(Y), \mathbb{Q} / \mathbb{Z}(j)) \stackrel{\partial_{y}}{\rightarrow} H_{y}^{*+1}(Y, \mathbb{Q} / \mathbb{Z}(j))
$$

is trivial. 
(2) If $x \in X^{(1)}$, the diagram

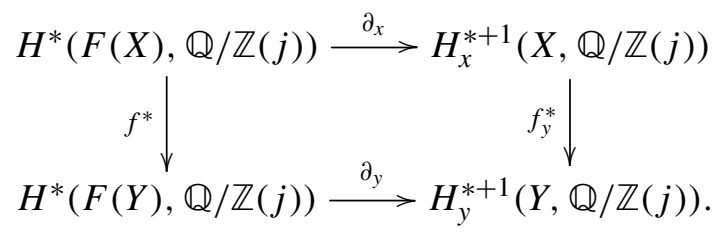

is commutative.

Lemma A.7. Let $X$ be a geometrically irreducible variety, $Z \subset X$ a closed subvariety of codimension 1 , and $x$ the generic point of $Z$. Let $P$ be a variety over $F$ such that $P(K)$ is dense in $P$ for every field extension $K / F$ with $K$ infinite, and let $y$ be the generic point of $Z \times P$ in $Y:=X \times P$. Then the homomorphism $f_{y}^{*}: H_{x}^{*}(X, \mathbb{Q} / \mathbb{Z}(j)) \rightarrow H_{y}^{*}(Y, \mathbb{Q} / \mathbb{Z}(j))$ induced by the projection $f: Y \rightarrow X$ is injective.

Proof. Assume first that the field $F$ is infinite. An element $\alpha \in H_{x}^{*}(X, \mathbb{Q} / \mathbb{Z}(j))$ is represented by an element $h \in H_{Z \cap U}^{*}(U, \mathbb{Q} / \mathbb{Z}(j))$ for a nonempty open set $U \subset X$ containing $x$. If $\alpha$ belongs to the kernel of

$$
f_{y}^{*}: H_{x}^{*}(X, \mathbb{Q} / \mathbb{Z}(j)) \rightarrow H_{y}^{*}(Y, \mathbb{Q} / \mathbb{Z}(j)),
$$

then there is an open subset $W \subset U \times P$ containing $y$ such that $h$ belongs to the kernel of the composition

$g: H_{Z \cap U}^{*}(U, \mathbb{Q} / \mathbb{Z}(j)) \rightarrow H_{(Z \cap U) \times P}^{*}(U \times P, \mathbb{Q} / \mathbb{Z}(j)) \rightarrow H_{(Z \times P) \cap W}^{*}(W, \mathbb{Q} / \mathbb{Z}(j))$.

As $F$ is infinite, by the assumption on $P$, there is a rational point $t \in P$ in the image of the dominant composition $(Z \times P) \cap W \hookrightarrow Z \times P \rightarrow P$. We have $(U \times t) \cap W=U^{\prime} \times t$ for an open subset $U^{\prime} \subset U$ such that $x \in U^{\prime}$. Composing $g$ with the homomorphism $H_{(Z \times P) \cap W}^{*}(W, \mathbb{Q} / \mathbb{Z}(j)) \rightarrow H_{Z \cap U^{\prime}}^{*}\left(U^{\prime}, \mathbb{Q} / \mathbb{Z}(j)\right)$ induced by the morphism $\left(U^{\prime}, Z \cap U^{\prime}\right) \rightarrow(W,(Z \times P) \cap W), u \mapsto(u, t)$, we see that $h$ belongs to the kernel of the restriction homomorphism $H_{Z \cap U}^{*}(U, \mathbb{Q} / \mathbb{Z}(j)) \rightarrow H_{Z \cap U^{\prime}}^{*}\left(U^{\prime}, \mathbb{Q} / \mathbb{Z}(j)\right)$, hence the image of $\alpha$ in $H_{x}^{*}(X, \mathbb{Q} / \mathbb{Z}(j))$ is trivial.

Suppose now that $F$ is a finite field. Choose a prime integer $p$ and an infinite algebraic pro- $p$-extension $L / F$. By the first part of the proof, the statement holds for the variety $X_{L}$ over $L$. By the restriction-corestriction $\operatorname{argument} \operatorname{Ker}\left(f_{y}^{*}\right)$ is a $p$-primary torsion group. Since this holds for every prime $p$, we have $\operatorname{Ker}\left(f_{y}^{*}\right)=0$.

Corollary A.8. Let $G$ be a linear algebraic group over $F$, let $E \rightarrow X$ be a $G$ torsor over a geometrically irreducible variety $X$ with $E$ a $G$-rational variety and consider the first projection $p: E^{2} / G \rightarrow X$. Let $x \in X$ and $y \in E^{2} / G$ be points of 
codimension 1 such that $p(y)=x$. Then the homomorphism

$$
p_{y}^{*}: H_{x}^{*}(X, \mathbb{Q} / \mathbb{Z}(j)) \rightarrow H_{y}^{*}\left(E^{2} / G, \mathbb{Q} / \mathbb{Z}(j)\right)
$$

is injective.

Proof. Choose a linear $G$-space $V$ and a nonempty $G$-variety $U$ that is $G$-isomorphic to open subschemes of $E$ and $V$. We can replace the variety $E^{2} / G$ by $(E \times U) / G$, an open subscheme in the vector bundle $(E \times V) / G$ over $X$. Shrinking $X$ around $x$, we may assume that the vector bundle is trivial, that is, $(E \times U) / G$ is isomorphic to an open subscheme in $X \times V$. The statement then follows from Lemma A.7. $\square$

Proposition A.9. In the conditions of Corollary A.8, let $h \in H^{*}(F(X), \mathbb{Q} / \mathbb{Z}(j))_{\text {bal. }}$ Then $\partial_{x}(h)=0$ for every point $x \in X$ of codimension 1 .

Proof. Let $y \in E^{2} / G$ be the point of codimension 1 such that $p_{1}(y)=x$. As $p_{2}(y)$ is the generic point of $X$, by Lemma A.6(1), $\partial_{y}\left(h^{\prime}\right)=0$, where $h^{\prime}=p_{1}^{*}(h)=$ $p_{2}^{*}(h)$ in $H^{*}\left(F\left(E^{2} / G\right), \mathbb{Q} / \mathbb{Z}(j)\right)$. It follows from Lemma A.6(2) that $\partial_{x}(h)$ is in the kernel of $\left(p_{1}\right)_{y}^{*}: H_{x}^{*}(X, \mathbb{Q} / \mathbb{Z}(j)) \rightarrow H_{y}^{*}\left(E^{2} / G, \mathbb{Q} / \mathbb{Z}(j)\right)$ and hence is trivial by Corollary A.8.

The sheaf $\mathscr{H}^{*}(\mathbb{Q} / \mathbb{Z}(j))$ defined in Section 3 has a flasque resolution related to the Cousin complex by [Colliot-Thélène et al. 1997, §2] (for the $p$-components with $p \neq \operatorname{char} F$ ) and [Gros and Suwa 1988, Theorem 1.4] (for the $p$-component with $p=\operatorname{char} F>0$ ):

$$
0 \rightarrow \mathscr{H}^{n}(\mathbb{Q} / \mathbb{Z}(j)) \rightarrow \coprod_{x \in X^{(0)}} i_{x *} H_{x}^{n}(X, \mathbb{Q} / \mathbb{Z}(j)) \rightarrow \coprod_{x \in X^{(1)}} i_{x *} H_{x}^{n+1}(X, \mathbb{Q} / \mathbb{Z}(j)) \rightarrow \cdots,
$$

where $i_{x}: \operatorname{Spec} F(x) \rightarrow X$ are the canonical morphisms. In particular, we have:

Proposition A.10. Let $X$ be a smooth variety over $F$. The sequence

$$
0 \rightarrow H_{\mathrm{Zar}}^{0}\left(X, \mathscr{H}^{*}(\mathbb{Q} / \mathbb{Z}(j))\right) \rightarrow H^{*}(F(X), \mathbb{Q} / \mathbb{Z}(j)) \stackrel{\partial}{\rightarrow} \coprod_{x \in X^{(1)}} H_{x}^{*+1}(X, \mathbb{Q} / \mathbb{Z}(j)),
$$

where $\partial=\bigsqcup \partial_{x}$, is exact.

Proposition A.11. Let $X$ be a smooth variety over $F$ and $x \in X$. The sequence

$$
\left.0 \rightarrow H^{*}\left(O_{X, x}, \mathbb{Q} / \mathbb{Z}(j)\right)\right) \rightarrow H^{*}(F(X), \mathbb{Q} / \mathbb{Z}(j)) \stackrel{\partial}{\rightarrow} \coprod_{\substack{x^{\prime} \in X^{(1)} \\ x^{\prime} \in\{x\}}} H_{x^{\prime}}^{*+1}(X, \mathbb{Q} / \mathbb{Z}(j))
$$

is exact. 


\section{Appendix B: Spectral sequences}

\section{B-I: Hochschild-Serre spectral sequence. Let}

$$
\mathscr{A} \stackrel{W}{\rightarrow} \mathscr{B} \stackrel{V}{\rightarrow} \mathscr{C}
$$

be additive left exact functors between abelian categories with enough injective objects. If $W$ takes injective objects to $V$-acyclic ones, there is a spectral sequence

$$
E_{2}^{p, q}=R^{p} V\left(R^{q} W(A)\right) \Rightarrow R^{p+q}(V W)(A)
$$

for every complex $A$ in $\mathscr{A}$ bounded from below.

We have exact triangles in the derived category of $\mathscr{B}$ :

$$
\begin{gathered}
\tau_{\leq n} R W(A) \rightarrow R W(A) \rightarrow \tau_{\geq n+1} R W(A) \rightarrow \tau_{\leq n} R W(A)[1], \\
\tau_{\leq n-1} R W(A) \rightarrow \tau_{\leq n} R W(A) \rightarrow R^{n} W(A)[-n] \rightarrow \tau_{\leq n-1} R W(A)[1] .
\end{gathered}
$$

The filtration on $R^{n}(V W)(A)$ is defined by

$$
F^{j} R^{n}(V W)(A)=\operatorname{Im}\left(R^{n} V\left(\tau_{\leq(n-j)} R W(A)\right) \rightarrow R^{n} V(R W(A))=R^{n}(V W)(A)\right) .
$$

As $\tau_{\geq n+1} R W(A)$ is acyclic in degrees less than or equal to $n$, the morphism

$$
R^{n} V\left(\tau_{\leq n} R W(A)\right) \rightarrow R^{n} V(R W(A))=R^{n}(V W)(A)
$$

is an isomorphism, in particular, $F^{0} R^{n}(V W)(A)=R^{n}(V W)(A)$.

The edge homomorphism is defined as the composition

$$
R^{n}(V W)(A) \stackrel{\sim}{\rightarrow} R^{n} V\left(\tau_{\leq n} R W(A)\right) \rightarrow R^{n} V\left(R^{n} W(A)[-n]\right)=V\left(R^{n} W(A)\right) .
$$

Moreover, the kernel $F^{1} R^{n}(V W)(A)$ of the edge homomorphism is isomorphic to $R^{n} V\left(\tau_{\leq n-1} R W(A)\right)$. We define the morphism $d_{n}$ as the composition

$d_{n}: F^{1} R^{n}(V W)(A) \rightarrow R^{n} V\left(R^{n-1} W(A)[-n+1]\right)=R^{1} V\left(R^{n-1} W(A)\right)=E_{2}^{1, n-1}$.

B-II: First spectral sequence. Let $X$ be a smooth variety over a field $F$. We have the functors

$$
\operatorname{Sheaves}_{\text {ét }}(X) \stackrel{q_{*}}{\rightarrow} \operatorname{Sheaves}_{\text {ét }}(F) \stackrel{V}{\rightarrow} A b,
$$

where $q_{*}$ is the push-forward map for the structure morphism $q: X \rightarrow \operatorname{Spec}(F)$ and $V(M)=H^{0}(F, M)$.

Consider the Hochschild-Serre spectral sequence

$$
E_{2}^{p, q}=H^{p}\left(F, H^{q}\left(X_{\mathrm{sep}}, \mathbb{Z}(2)\right) \Rightarrow H^{p+q}(X, \mathbb{Z}(2)) .\right.
$$

Set $\Delta(i):=R q_{*}(\mathbb{Z}(i))$ for $i=1$ or 2 . Then $\Delta(i)$ is the complex of étale sheaves on $F$ concentrated in degrees $\geq 1$. The $j$-th term $F^{j} H^{n}(X, \mathbb{Z}(i))$ of the filtration 
on $H^{n}(X, \mathbb{Z}(i))$ coincides with the image of the canonical homomorphism

$$
H^{n}\left(F, \tau_{\leq(n-j)} \Delta(i)\right) \rightarrow H^{n}(F, \Delta(i))=H^{n}(X, \mathbb{Z}(i)) .
$$

Let $M$ be a $\Gamma$-lattice viewed as an étale sheaf over $F$. Note that there are canonical isomorphisms

$$
H^{*}\left(F, M^{\circ} \otimes \Delta(i)\right)=\operatorname{Ext}_{F}^{*}(M, \Delta(i))=\operatorname{Ext}_{X}^{*}\left(q^{*} M, \mathbb{Z}(i)\right),
$$

where $M^{\circ}:=\operatorname{Hom}(M, \mathbb{Z})$ is the dual lattice.

Consider also the following product map:

$$
\mathbb{Z}(1) \otimes^{L} \Delta(1) \rightarrow R q_{*}\left(q^{*} \mathbb{Z}(1) \otimes^{L} \mathbb{Z}(1)\right) \rightarrow R q_{*}\left(\mathbb{Z}(1) \otimes^{L} \mathbb{Z}(1)\right) \rightarrow R q_{*}(\mathbb{Z}(2)) .
$$

The complex $\mathbb{Z}(1) \otimes{ }^{L} \tau_{\leq 2} \Delta(1)$ is trivial in degrees greater than 3 , hence we have a commutative diagram

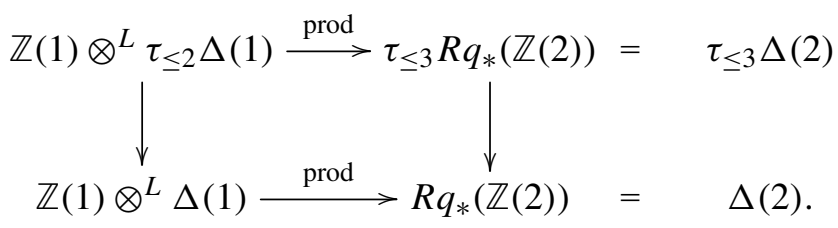

There are canonical morphisms from (B-2):

$$
h_{2}: \tau_{\leq 2} \Delta(1)[2] \rightarrow H^{2}\left(X_{\text {sep }}, \mathbb{Z}(1)\right) \quad \text { and } \quad h_{3}: \tau_{\leq 3} \Delta(2)[3] \rightarrow H^{3}\left(X_{\text {sep }}, \mathbb{Z}(2)\right) .
$$

Consider an element

$$
\delta \in H^{1}\left(F, M \otimes F_{\text {sep }}^{\times}\right)=\operatorname{Ext}_{F}^{1}\left(M^{\circ}, \mathbb{G}_{m, F}\right)=\operatorname{Ext}_{F}^{2}\left(M^{\circ}, \mathbb{Z}(1)\right),
$$

and view $\delta$ as a morphism $\delta: M^{\circ} \rightarrow \mathbb{Z}(1)[2]$ in $D^{+}$(Sheavesét $(F)$ ).

The following diagram

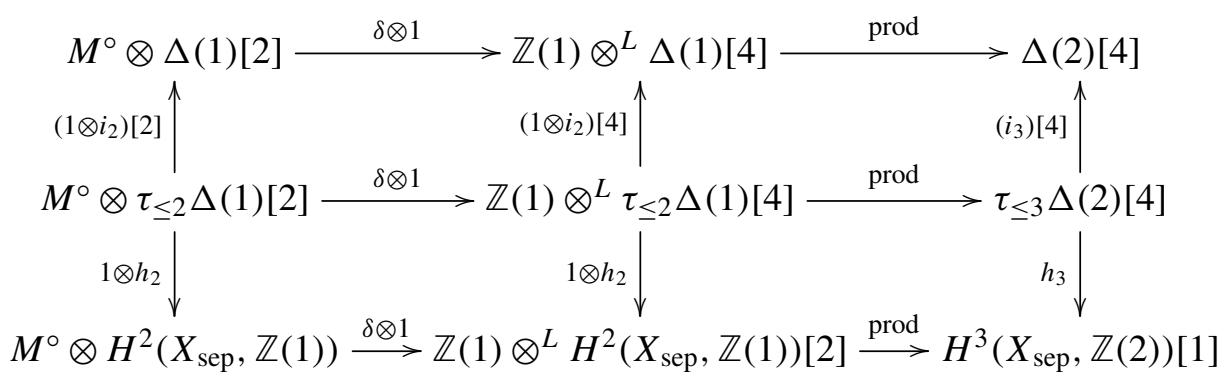

where $i_{2}: \tau_{\leq 2} \Delta(1) \rightarrow \Delta(1)$ and $i_{3}: \tau_{\leq 3} \Delta(2) \rightarrow \Delta(2)$ are natural morphisms, is commutative.

By (B-4), we have

$$
H^{0}\left(F, M^{\circ} \otimes \Delta(1)[2]\right)=\operatorname{Ext}_{F}^{2}(M, \Delta(1))=\operatorname{Ext}_{X}^{2}\left(q^{*} M, \mathbb{Z}(1)\right) .
$$


Furthermore, the diagram above yields a commutative square

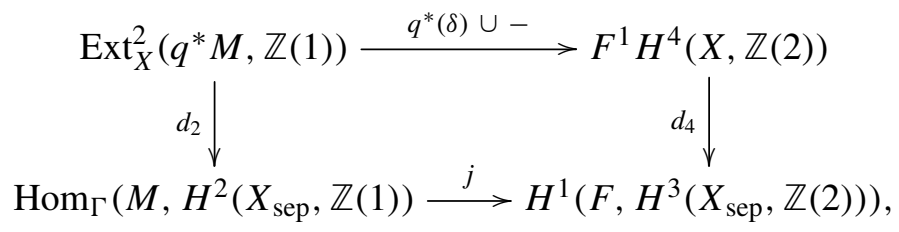

where $d_{2}$ is the edge map coming from the spectral sequence

$$
\operatorname{Ext}_{F}^{p}\left(M, H^{q}\left(X_{\text {sep }}, \mathbb{Z}(1)\right)\right) \Rightarrow \operatorname{Ext}_{X}^{p+q}\left(q^{*} M, \mathbb{Z}(1)\right)
$$

and $j$ coincides with the composition

$$
\begin{aligned}
\operatorname{Hom}_{\Gamma}\left(M, H^{2}\left(X_{\mathrm{sep}}, \mathbb{Z}(1)\right)=H^{0}\left(F, M^{\circ} \otimes H^{2}\left(X_{\mathrm{sep}}, \mathbb{Z}(1)\right)\right)\right. \\
\stackrel{\delta \cup-}{\longrightarrow} H^{1}\left(F, F_{\mathrm{sep}}^{\times} \otimes H^{2}\left(X_{\mathrm{sep}}, \mathbb{Z}(1)\right)\right) \stackrel{\rho}{\rightarrow} H^{1}\left(F, H^{3}\left(X_{\mathrm{sep}}, \mathbb{Z}(2)\right)\right),
\end{aligned}
$$

with $\rho$ given by the product map.

Now suppose the group $H^{2}\left(X_{\text {sep }}, \mathbb{Z}(1)\right)$, which is canonically isomorphic to $\operatorname{Pic}\left(X_{\text {sep }}\right)$, is a lattice. Let $M=\operatorname{Pic}\left(X_{\text {sep }}\right)$ and consider the torus $T$ over $F$ with $\widehat{T}_{\text {sep }}=M$. It follows that

$$
\delta \in H^{1}\left(F, T^{\circ}\right)=H^{1}\left(F, \widehat{T}_{\text {sep }} \otimes F_{\text {sep }}^{\times}\right)=H^{2}\left(F, \widehat{T}_{\text {sep }} \otimes \mathbb{Z}(1)\right),
$$

where $T^{\circ}$ is the dual torus. Note that $\delta \cup 1_{M}=\delta$, where

$$
1_{M} \in H^{0}\left(F, M^{\circ} \otimes H^{2}\left(X_{\mathrm{sep}}, \mathbb{Z}(1)\right)\right)=\operatorname{End}_{\Gamma}(M)
$$

is the identity.

The top map in the last diagram is given by the pairing

$$
\begin{aligned}
H^{1}\left(X, T^{0}\right) \otimes H^{1}(X, T) & \rightarrow F^{1} H^{4}(X, \mathbb{Z}(2)), \\
a \otimes b & \mapsto a \cup b,
\end{aligned}
$$

defined as the cup-product in (4-3),

$$
H^{2}(X, \widehat{T}(1)) \otimes H^{2}\left(X, \widehat{T}^{\circ}(1)\right) \rightarrow F^{1} H^{4}(X, \mathbb{Z}(2)),
$$

if we identify $\operatorname{Ext}_{X}^{2}\left(q^{*} M, \mathbb{Z}(1)\right)$ with $H^{2}\left(X, \widehat{T}^{\circ}(1)\right)=H^{1}(X, T)$.

In this case, the homomorphism

$$
\rho: H^{1}\left(F, T^{\circ}\right) \rightarrow H^{1}\left(F, H^{3}\left(X_{\mathrm{sep}}, \mathbb{Z}(2)\right)\right)
$$

is given by the product homomorphism

$$
T^{\circ}\left(F_{\text {sep }}\right)=F_{\text {sep }}^{\times} \otimes \widehat{T}_{\text {sep }}=F_{\text {sep }}^{\times} \otimes \operatorname{Pic}\left(X_{\text {sep }}\right) \rightarrow H^{3}\left(X_{\text {sep }}, \mathbb{Z}(2)\right) .
$$


A $T$-torsor $E \rightarrow X$ is called universal if the class of $E$ in

$$
H^{1}(X, T)=\operatorname{Ext}_{X}^{2}\left(q^{*} M, \mathbb{Z}(1)\right)
$$

satisfies $d_{2}([E])=1_{M}$; see [Colliot-Thélène and Sansuc 1987a].

Commutativity of the previous diagram gives:

Proposition B.1. Let $X$ be a smooth variety over $F$ such that $\operatorname{Pic}\left(X_{\mathrm{sep}}\right)$ is a lattice. Let $T$ be the torus over $F$ satisfying $\widehat{T}_{\mathrm{sep}}=\operatorname{Pic}\left(X_{\mathrm{sep}}\right)$ and let $E$ be a universal $T$-torsor over $X$ with the class $[E] \in H^{1}(X, T)$. Then for every $\delta \in H^{1}\left(F, T^{\circ}\right)$, we have

$$
d_{4}\left(q^{*}(\delta) \cup[E]\right)=\rho(\delta),
$$

where $d_{4}: F^{1} H^{4}(X, \mathbb{Z}(2)) \rightarrow H^{1}\left(F, H^{3}\left(X_{\mathrm{sep}}, \mathbb{Z}(2)\right)\right)$ is the map induced by the Hochschild-Serre spectral sequence (B-3) and the cup-product is taken for the pairing (B-6).

B-III: Second spectral sequence. We assume that $H^{3}\left(X_{\mathrm{sep}}, \mathbb{Z}(2)\right)=0$, hence in particular $E_{2}^{0,3}=0$ in the spectral sequence (B-3) and so $E_{\infty}^{2,2} \subset E_{2}^{2,2}$. Therefore, we have a canonical map

$$
e_{4}: F^{2} H^{4}(X, \mathbb{Z}(2)) \rightarrow E_{\infty}^{2,2} \hookrightarrow E_{2}^{2,2}=H^{2}\left(F, H^{2}\left(X_{\mathrm{sep}}, \mathbb{Z}(2)\right) .\right.
$$

Let $N$ be a $\Gamma$-lattice. Consider an element

$$
\gamma \in H^{2}\left(F, N \otimes F_{\text {sep }}^{\times}\right)=\operatorname{Ext}_{F}^{2}\left(N^{\circ}, \mathbb{G}_{m, F}\right)=\operatorname{Ext}_{F}^{3}\left(N^{\circ}, \mathbb{Z}(1)\right),
$$

and view $\gamma$ as a morphism $\gamma: N^{\circ} \rightarrow \mathbb{Z}(1)[3]$ in $D^{+}$(Sheavesét $\left.(F)\right)$.

As above, the commutative diagram

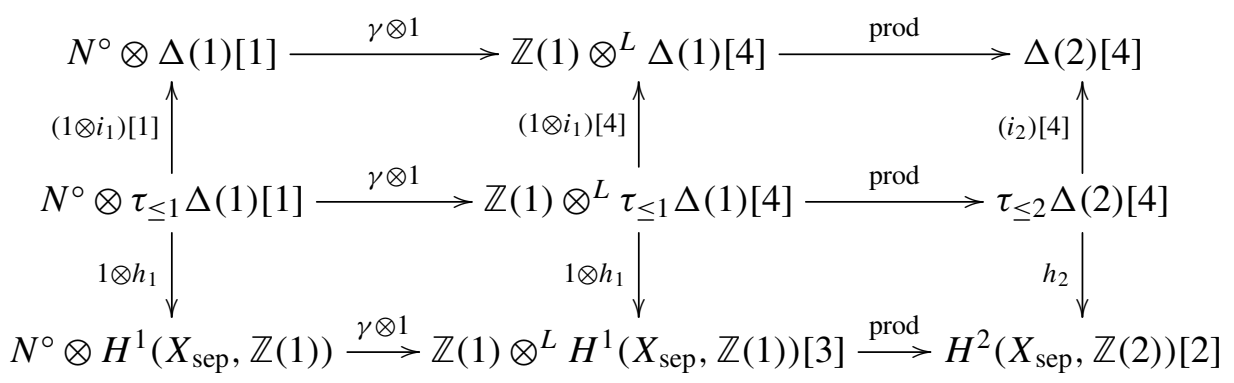

where $i_{1}, i_{2}, h_{1}$ and $h_{2}$ are defined in a similar fashion as in Section B-II, yields a commutative square

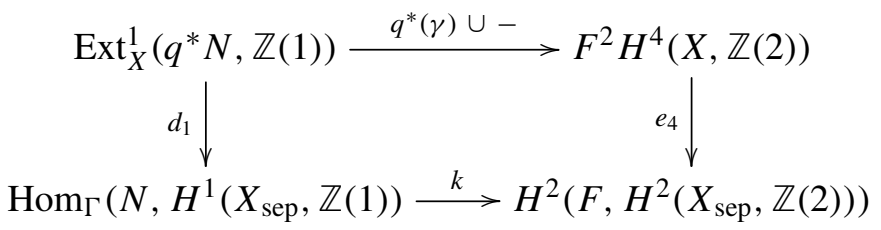


where $d_{1}$ is the edge map coming from the spectral sequence

$$
\operatorname{Ext}_{F}^{p}\left(N, H^{q}\left(X_{\text {sep }}, \mathbb{Z}(1)\right)\right) \Rightarrow \operatorname{Ext}_{X}^{p+q}\left(q^{*} N, \mathbb{Z}(1)\right)
$$

and $k$ coincides with the composition

$$
\begin{aligned}
& \operatorname{Hom}_{\Gamma}\left(N, H^{1}\left(X_{\text {sep }}, \mathbb{Z}(1)\right)=H^{0}\left(F, N^{\circ} \otimes H^{1}\left(X_{\text {sep }}, \mathbb{Z}(1)\right)\right)\right. \\
& \stackrel{\gamma \cup-}{\longrightarrow} H^{2}\left(F, F_{\text {sep }}^{\times} \otimes H^{1}\left(X_{\text {sep }}, \mathbb{Z}(1)\right)\right) \rightarrow H^{2}\left(F, H^{2}\left(X_{\text {sep }}, \mathbb{Z}(2)\right)\right)
\end{aligned}
$$

with the last homomorphism given by the product map.

Suppose $N$ is a $\Gamma$-lattice in $F_{\text {sep }}[X]^{\times}$such that the composition

$$
N \hookrightarrow F_{\text {sep }}[X]^{\times} \rightarrow F_{\text {sep }}[X]^{\times} / F_{\text {sep }}^{\times}
$$

is an isomorphism. Consider the torus $Q$ with $\widehat{Q}_{\text {sep }}=N$, so that $\gamma \in H^{2}\left(F, Q^{\circ}\right)$.

Note that $\gamma \cup i_{N}=\gamma$, where

$$
i_{N} \in H^{0}\left(F, N^{\circ} \otimes H^{1}\left(X_{\mathrm{sep}}, \mathbb{Z}(1)\right)\right)=\operatorname{Hom}_{\Gamma}\left(N, F_{\mathrm{sep}}[X]^{\times}\right)
$$

is the embedding.

The top map in the previous diagram is given by the pairing

$$
\begin{aligned}
H^{2}\left(X, Q^{0}\right) \otimes H^{0}(X, Q) & \rightarrow F^{2} H^{4}(X, \mathbb{Z}(2)), \\
a \otimes b & \mapsto a \cup b,
\end{aligned}
$$

defined as the cup-product in (4-3),

$$
\left.H^{3}(X, \widehat{Q}(1)) \otimes H^{1}\left(X, \widehat{Q}^{\circ}(1)\right)\right) \rightarrow H^{4}(X, \mathbb{Z}(2)),
$$

if we identify $\operatorname{Ext}_{X}^{1}\left(q^{*} N, \mathbb{Z}(1)\right)$ with $H^{1}\left(X, \widehat{Q}^{\circ}(1)\right)=H^{0}(X, Q)$.

The inclusion of $\widehat{Q}_{\text {sep }}$ into $F_{\text {sep }}[X]^{\times}$yields a morphism $\varepsilon: X \rightarrow Q$ that can be viewed as an element of $H^{0}(X, Q)$. Consider the map

$$
\mu: H^{2}\left(F, Q^{\circ}\right) \rightarrow H^{2}\left(F, H^{2}\left(X_{\text {sep }}, \mathbb{Z}(2)\right)\right)
$$

given by composition with the product homomorphism

$$
Q^{\circ}\left(F_{\text {sep }}\right)=F_{\text {sep }}^{\times} \otimes \widehat{Q}_{\text {sep }} \rightarrow F_{\text {sep }}^{\times} \otimes H^{1}\left(X_{\text {sep }}, \mathbb{Z}(1)\right) \rightarrow H^{2}\left(X_{\text {sep }}, \mathbb{Z}(2)\right) .
$$

We have proved:

Proposition B.2. Let $X$ be a smooth variety over $F$ such that $H^{3}\left(X_{\mathrm{sep}}, \mathbb{Z}(2)\right)=0$. Let $N$ be a $\Gamma$-lattice in $F_{\mathrm{sep}}[X]^{\times}$such that the composition

$$
N \hookrightarrow F_{\text {sep }}[X]^{\times} \rightarrow F_{\text {sep }}[X]^{\times} / F_{\text {sep }}^{\times}
$$


is an isomorphism. Let $Q$ be the torus over $F$ satisfying $\widehat{Q}_{\text {sep }}=N$. Then for every $\gamma \in H^{2}\left(F, Q^{\circ}\right)$, we have

$$
e_{4}\left(q^{*}(\gamma) \cup \varepsilon\right)=\mu(\gamma)
$$

where $e_{4}: F^{2} H^{4}(X, \mathbb{Z}(2)) \rightarrow H^{2}\left(F, H^{2}\left(X_{\mathrm{sep}}, \mathbb{Z}(2)\right)\right)$ is the map induced by the Hochschild-Serre spectral sequence (B-3) and the cup-product is taken for the pairing (B-8).

B-IV: Relative étale cohomology. Let $X$ be a smooth variety over $F$. Following B. Kahn [1996, §3], we define the relative étale cohomology groups as follows. Recall that $\Delta(i)=R q_{*}(\mathbb{Z}(i))$ for $i=1$ and 2 , where $q: X \rightarrow \operatorname{Spec}(F)$ is the structure morphism, and let $\Delta^{\prime}(i)$ be the cone of the natural morphism $\mathbb{Z}(i) \rightarrow \Delta(i)$ in $D_{+}($Sheavesét $(F))$. Define

$$
H^{*}(X / F, \mathbb{Z}(2)):=H^{*}\left(F, \Delta^{\prime}(2)\right) .
$$

(Note that our indexing is different from that in [Kahn 1996, §3].)

There is an infinite exact sequence

$$
\cdots \rightarrow H^{i}(F, \mathbb{Z}(2)) \rightarrow H^{i}(X, \mathbb{Z}(2)) \rightarrow H^{i}(X / F, \mathbb{Z}(2)) \rightarrow H^{i+1}(F, \mathbb{Z}(2)) \rightarrow \cdots
$$

If $X$ has a rational point, we have

$$
H^{i}(X / F, \mathbb{Z}(2))=\bar{H}^{i}(X, \mathbb{Z}(2)):=H^{i}(X, \mathbb{Z}(2)) / H^{i}(F, \mathbb{Z}(2)) .
$$

There is a Hochschild-Serre type spectral sequence [Kahn 1996, §3]

$$
E_{2}^{p, q}=H^{p}\left(F, H^{q}\left(X_{\mathrm{sep}} / F_{\mathrm{sep}}, \mathbb{Z}(2)\right)\right) \Rightarrow H^{p+q}(X / F, \mathbb{Z}(2)),
$$

and we have by [Kahn 1996, Lemma 3.1] that

$$
H^{q}\left(X_{\mathrm{sep}} / F_{\mathrm{sep}}, \mathbb{Z}(2)\right)= \begin{cases}0 & \text { if } q \leq 0, \\ \text { uniquely divisible group } & \text { if } q=1, \\ \bar{H}_{\mathrm{Zar}}^{0}\left(X_{\mathrm{sep}}, \mathscr{K}_{2}\right) & \text { if } q=2, \\ H_{\mathrm{Zar}}^{1}\left(X_{\mathrm{sep}}, \mathscr{K}_{2}\right) & \text { if } q=3 .\end{cases}
$$

It follows that $E_{2}^{p, q}=0$ if $q \leq 1$ and $p>0$. Comparing the spectral sequences (B-3) and (B-10), by Proposition B.1 we have:

Proposition B.3. Let $X$ be a smooth variety over $F$ such that $X(F) \neq \varnothing$. If $H_{\mathrm{Zar}}^{0}\left(X_{\mathrm{sep}}, \mathscr{K}_{2}\right)=K_{2}\left(F_{\mathrm{sep}}\right)$, then the spectral sequence (B-10) yields an exact sequence

$$
\begin{aligned}
0 \rightarrow H^{1}\left(F, H_{\text {Zar }}^{1}\left(X_{\text {sep }}, \mathscr{K}_{2}\right)\right) \stackrel{\alpha}{\rightarrow} & \bar{H}^{4}(X, \mathbb{Z}(2)) \\
& \rightarrow \bar{H}^{4}\left(X_{\text {sep }}, \mathbb{Z}(2)\right)^{\Gamma} \rightarrow H^{2}\left(F, H_{\text {Zar }}^{1}\left(X_{\text {sep }}, \mathscr{K}_{2}\right)\right) .
\end{aligned}
$$


If, moreover, the group $\operatorname{Pic}\left(X_{\mathrm{sep}}\right)$ is a lattice and $T$ is the torus over $F$ such that $\widehat{T}_{\text {sep }}=\operatorname{Pic}\left(X_{\text {sep }}\right)$, then $\alpha(\rho(\delta))=q^{*}(\delta) \cup[E]$ for every $\delta \in H^{1}\left(F, T^{\circ}\right)$, where $\rho$ is defined in (B-7) and $E$ is a universal $T$-torsor over $X$.

Comparing the spectral sequences (B-3) and (B-10), by Proposition B.2 we have: Proposition B.4. Let $X$ be a smooth variety over $F$ such that $X(F) \neq \varnothing$. If $H_{\mathrm{Zar}}^{1}\left(X_{\mathrm{sep}}, \mathscr{K}_{2}\right)=0$, then the spectral sequence (B-10) yields an exact sequence

$$
\begin{aligned}
0 \rightarrow H^{2}\left(F, \bar{H}_{\mathrm{Zar}}^{0}\left(X_{\mathrm{sep}}, \mathscr{K}_{2}\right)\right) \stackrel{\beta}{\rightarrow} & \bar{H}^{4}(X, \mathbb{Z}(2)) \\
& \rightarrow \bar{H}^{4}\left(X_{\mathrm{sep}}, \mathbb{Z}(2)\right)^{\Gamma} \rightarrow H^{3}\left(F, \bar{H}_{\mathrm{Zar}}^{0}\left(X_{\mathrm{sep}}, \mathscr{K}_{2}\right)\right) .
\end{aligned}
$$

If $N$ is a $\Gamma$-lattice in $F_{\text {sep }}[X]^{\times}$such that the composition

$$
N \hookrightarrow F_{\mathrm{sep}}[X]^{\times} \rightarrow F_{\mathrm{sep}}[X]^{\times} / F_{\text {sep }}^{\times}
$$

is an isomorphism and $Q$ is the torus over $F$ satisfying $\widehat{Q}_{\text {sep }}=N$, then $\beta(\mu(\gamma))=$ $q^{*}(\gamma) \cup \varepsilon$ for every $\gamma \in H^{2}\left(F, Q^{\circ}\right)$, where $\mu$ is defined in (B-9) and $\varepsilon \in H^{0}(X, Q)$ is given by the inclusion of $\widehat{Q}_{\text {sep }}$ into $F_{\text {sep }}[X]^{\times}$.

\section{Acknowledgements}

The authors would like to thank Jean-Louis Colliot-Thélène, Skip Garibaldi, Thomas Geisser, David Harari, Bruno Kahn, Kazuya Kato, Alena Pirutka and the referee for helpful discussions and comments.

\section{References}

[Bloch and Kato 1986] S. Bloch and K. Kato, "p-adic étale cohomology", Inst. Hautes Études Sci. Publ. Math. 63 (1986), 107-152. MR 87k:14018 Zbl 0613.14017

[Bogomolov 1987] F. A. Bogomolov, "The Brauer group of quotient spaces of linear representations”, Izv. Akad. Nauk SSSR Ser. Mat. 51:3 (1987), 485-516, 688. In Russian; translated in Math. USSR-Izv. 30:3 (1988), 455-485. MR 88m:16006 Zbl 0641.14005

[Cartan and Eilenberg 1999] H. Cartan and S. Eilenberg, Homological algebra, Princeton Landmarks in Mathematics, Princeton University Press, 1999. MR 2000h:18022 Zbl 0933.18001

[Colliot-Thélène 1995] J.-L. Colliot-Thélène, "Birational invariants, purity and the Gersten conjecture", pp. 1-64 in $K$-theory and algebraic geometry: connections with quadratic forms and division algebras (Santa Barbara, CA, 1992), edited by B. Jacob and A. Rosenberg, Proc. Sympos. Pure Math. 58, Amer. Math. Soc., Providence, RI, 1995. MR 96c:14016 Zbl 0834.14009

[Colliot-Thélène 2004] J.-L. Colliot-Thélène, "Résolutions flasques des groupes réductifs connexes", C. R. Math. Acad. Sci. Paris 339:5 (2004), 331-334. MR 2005f:20081 Zbl 1076.20034

[Colliot-Thélène and Ojanguren 1989] J.-L. Colliot-Thélène and M. Ojanguren, "Variétés unirationnelles non rationnelles: Au-delà de l'exemple d'Artin et Mumford", Invent. Math. 97:1 (1989), 141-158. MR 90m:14012 Zbl 0686.14050

[Colliot-Thélène and Sansuc 1977] J.-L. Colliot-Thélène and J.-J. Sansuc, "La $R$-équivalence sur les tores”, Ann. Sci. École Norm. Sup. (4) 10:2 (1977), 175-229. MR 56 \#8576 Zbl 0356.14007 
[Colliot-Thélène and Sansuc 1987a] J.-L. Colliot-Thélène and J.-J. Sansuc, "La descente sur les variétés rationnelles, II”, Duke Math. J. 54:2 (1987), 375-492. MR 89f:11082 Zbl 0659.14028

[Colliot-Thélène and Sansuc 1987b] J.-L. Colliot-Thélène and J.-J. Sansuc, "Principal homogeneous spaces under flasque tori: Applications", J. Algebra 106:1 (1987), 148-205. MR 88j:14059 Zbl 0597.14014

[Colliot-Thélène et al. 1997] J.-L. Colliot-Thélène, R. T. Hoobler, and B. Kahn, "The Bloch-OgusGabber theorem", pp. 31-94 in Algebraic K-theory (Toronto, ON, 1996), edited by V. P. Snaith, Fields Inst. Commun. 16, Amer. Math. Soc., Providence, RI, 1997. MR 98j:14021 Zbl 0911.14004

[Colliot-Thélène et al. 2005] J.-L. Colliot-Thélène, D. Harari, and A. N. Skorobogatov, "Compactification équivariante d'un tore (d'après Brylinski et Künnemann)", Expo. Math. 23:2 (2005), 161-170. MR 2006c:14076 Zbl 1078.14076

[Demazure and Grothendieck 1970] M. Demazure and A. Grothendieck (editors), Schémas en groupes, III: Structure des schémas en groupes réductifs. Exposés XIX à XXVI. (Séminaire de Géométrie Algébrique du Bois Marie 1962/64 (SGA 3)), Lecture Notes in Mathematics 153, Springer, Berlin, 1970. MR 43 \#223c Zbl 0212.52810

[Edidin and Graham 1998] D. Edidin and W. Graham, "Equivariant intersection theory", Invent. Math. 131:3 (1998), 595-634. MR 99j:14003a Zbl 0940.14003

[Esnault et al. 1998] H. Esnault, B. Kahn, M. Levine, and E. Viehweg, "The Arason invariant and mod 2 algebraic cycles", J. Amer. Math. Soc. 11:1 (1998), 73-118. MR 98d:14010 Zbl 1025.11009

[Fulton 1984] W. Fulton, Intersection theory, Ergebnisse der Mathematik und ihrer Grenzgebiete

(3) [Results in Mathematics and Related Areas (3)] 2, Springer, Berlin, 1984. MR 85k:14004 Zbl 0541.14005

[Garibaldi et al. 2003] S. Garibaldi, A. Merkurjev, and J.-P. Serre, Cohomological invariants in Galois cohomology, University Lecture Series 28, American Mathematical Society, Providence, RI, 2003. MR 2004f:11034 Zbl 1159.12311

[Gros 1985] M. Gros, Classes de Chern et classes de cycles en cohomologie de Hodge-Witt logarithmique, Mém. Soc. Math. France (N.S.) 21, Société Mathématique de France, Paris, 1985. MR 87m:14021 Zbl 0615.14011

[Gros and Suwa 1988] M. Gros and N. Suwa, "La conjecture de Gersten pour les faisceaux de Hodge-Witt logarithmique”, Duke Math. J. 57:2 (1988), 615-628. MR 89h:14006b Zbl 0715.14011

[Humphreys 1990] J. E. Humphreys, Reflection groups and Coxeter groups, Cambridge Studies in Advanced Mathematics 29, Cambridge University Press, 1990. MR 92h:20002 Zbl 0725.20028

[Illusie 1979] L. Illusie, "Complexe de de Rham-Witt et cohomologie cristalline", Ann. Sci. École Norm. Sup. (4) 12:4 (1979), 501-661. MR 82d:14013 Zbl 0436.14007

[Izhboldin 1991] O. Izhboldin, "On $p$-torsion in $K_{*}^{M}$ for fields of characteristic $p$ ", pp. 129-144 in Algebraic K-theory, edited by A. A. Suslin, Adv. Soviet Math. 4, Amer. Math. Soc., Providence, RI, 1991. MR 92f:11165 Zbl 0746.19002

[Kahn 1993] B. Kahn, "Descente galoisienne et $K_{2}$ des corps de nombres", K-Theory 7:1 (1993), 55-100. MR 94i:11094 Zbl 0780.12007

[Kahn 1996] B. Kahn, "Applications of weight-two motivic cohomology”, Doc. Math. 1:17 (1996), 395-416. MR 98b:14007 Zbl 0883.19002

[Knus et al. 1998] M.-A. Knus, A. Merkurjev, M. Rost, and J.-P. Tignol, The book of involutions, American Mathematical Society Colloquium Publications 44, American Mathematical Society, Providence, RI, 1998. MR 2000a:16031 Zbl 0955.16001

[Lang 1956] S. Lang, "Algebraic groups over finite fields", Amer. J. Math. 78 (1956), 555-563. MR 19,174a Zbl 0073.37901 
[Lichtenbaum 1987] S. Lichtenbaum, "The construction of weight-two arithmetic cohomology", Invent. Math. 88:1 (1987), 183-215. MR 88d:14011 Zbl 0615.14004

[Lichtenbaum 1990] S. Lichtenbaum, "New results on weight-two motivic cohomology", pp. 35-55 in The Grothendieck Festschrift, III, edited by P. Cartier et al., Progr. Math. 88, Birkhäuser, Boston, MA, 1990. MR 92m:14030 Zbl 0809.14004

[Merkurjev and Panin 1997] A. S. Merkurjev and I. A. Panin, " $K$-theory of algebraic tori and toric varieties”, K-Theory 12:2 (1997), 101-143. MR 98j:19005 Zbl 0882.19002

[Milne 1980] J. S. Milne, Étale cohomology, Princeton Mathematical Series 33, Princeton University Press, 1980. MR 81j:14002 Zbl 0433.14012

[Quillen 1973] D. Quillen, "Higher algebraic K-theory, I", pp. 85-147 in Algebraic K-theory, I: Higher K-theories (Seattle, Wash., 1972), edited by H. Bass, Lecture Notes in Math. 341, Springer, Berlin, 1973. MR 49 \#2895 Zbl 0292.18004

[Rost 1996] M. Rost, “Chow groups with coefficients”, Doc. Math. 1:16 (1996), 319-393. MR 98a: 14006 Zbl 0864.14002

[Sansuc 1981] J.-J. Sansuc, “Groupe de Brauer et arithmétique des groupes algébriques linéaires sur un corps de nombres", J. Reine Angew. Math. 327 (1981), 12-80. MR 83d:12010 Zbl 0468.14007

[Totaro 1999] B. Totaro, "The Chow ring of a classifying space", pp. 249-281 in Algebraic K-theory (Seattle, WA, 1997), edited by W. Raskind and C. Weibel, Proc. Sympos. Pure Math. 67, Amer. Math. Soc., Providence, RI, 1999. MR 2001f:14011 Zbl 0967.14005

[Voskresenskiǔ 1988] V. E. Voskresenski1̌, "Maximal tori without affect in semisimple algebraic groups", Mat. Zametki 44:3 (1988), 309-318, 410. MR 90a:11046

[Voskresenskiı̌ 1998] V. E. Voskresenskiü, Algebraic groups and their birational invariants, Translations of Mathematical Monographs 179, American Mathematical Society, Providence, RI, 1998. MR 99g:20090

Communicated by Jean-Louis Colliot-Thélène Received 2012-04-23 Revised 2012-10-08 Accepted 2012-11-09

blinstein@math.ucla.edu Department of Mathematics, University of California, Los Angeles, Los Angeles CA 90095-1555, United States

merkurev@math.ucla.edu Department of Mathematics, University of California, Los Angeles, Los Angeles CA 90095-1555, United States 


\section{Algebra \& Number Theory}

msp.org/ant

\section{EDITORS}

MANAGING EDITOR

Bjorn Poonen

Massachusetts Institute of Technology

Cambridge, USA

\author{
EDITORIAL BOARD CHAIR \\ David Eisenbud \\ University of California \\ Berkeley, USA
}

\section{BOARD OF EDITORS}

Georgia Benkart

Dave Benson

Richard E. Borcherds

John H. Coates

J-L. Colliot-Thélène

Brian D. Conrad

Hélène Esnault

Hubert Flenner

Edward Frenkel

Andrew Granville

Joseph Gubeladze

Roger Heath-Brown

Ehud Hrushovski

Craig Huneke

Mikhail Kapranov

Yujiro Kawamata

János Kollár

Yuri Manin

Barry Mazur

Philippe Michel
University of Wisconsin, Madison, USA

University of Aberdeen, Scotland

University of California, Berkeley, USA

University of Cambridge, UK

CNRS, Université Paris-Sud, France

University of Michigan, USA

Freie Universität Berlin, Germany

Ruhr-Universität, Germany

University of California, Berkeley, USA

Université de Montréal, Canada

San Francisco State University, USA

Oxford University, UK

Hebrew University, Israel

University of Virginia, USA

Yale University, USA

University of Tokyo, Japan

Princeton University, USA

Northwestern University, USA

Harvard University, USA

École Polytechnique Fédérale de Lausanne
Susan Montgomery

Shigefumi Mori

Raman Parimala

Jonathan Pila

Victor Reiner

Karl Rubin

Peter Sarnak

Joseph H. Silverman

Michael Singer

Vasudevan Srinivas

J. Toby Stafford

Bernd Sturmfels

Richard Taylor

Ravi Vakil

Michel van den Bergh

Marie-France Vignéras

Kei-Ichi Watanabe

Efim Zelmanov

Shou-Wu Zhang
University of Southern California, USA

RIMS, Kyoto University, Japan

Emory University, USA

University of Oxford, UK

University of Minnesota, USA

University of California, Irvine, USA

Princeton University, USA

Brown University, USA

North Carolina State University, USA

Tata Inst. of Fund. Research, India

University of Michigan, USA

University of California, Berkeley, USA

Harvard University, USA

Stanford University, USA

Hasselt University, Belgium

Université Paris VII, France

Nihon University, Japan

University of California, San Diego, USA

Princeton University, USA

PRODUCTION

production@msp.org

Silvio Levy, Scientific Editor

See inside back cover or msp.org/ant for submission instructions.

The subscription price for 2013 is US $\$ 200 /$ year for the electronic version, and $\$ 350 /$ year $(+\$ 40$, if shipping outside the US) for print and electronic. Subscriptions, requests for back issues and changes of subscribers address should be sent to MSP.

Algebra \& Number Theory (ISSN 1944-7833 electronic, 1937-0652 printed) at Mathematical Sciences Publishers, 798 Evans Hall \#3840, c/o University of California, Berkeley, CA 94720-3840 is published continuously online. Periodical rate postage paid at Berkeley, CA 94704, and additional mailing offices.

ANT peer review and production are managed by EditFLOW ${ }^{\circledR}$ from Mathematical Sciences Publishers.

\section{PUBLISHED BY}

- mathematical sciences publishers

nonprofit scientific publishing

http://msp.org/

(C) 2013 Mathematical Sciences Publishers 


\section{Algebra \& Number Theory}

$\begin{array}{lll}\text { Volume } 7 & \text { No. } 7 \quad 2013\end{array}$

Weil representation and transfer factor

TERUJI THOMAS

Analytic families of finite-slope Selmer groups

1571

JONATHAN POTTHARST

Multiplicative excellent families of elliptic surfaces of type $E_{7}$ or $E_{8}$

ABHINAV KUMAR and TETSUJI SHIODA

Cohomological invariants of algebraic tori

SAM Blinstein and AleXANDer MerkurJeV

On abstract representations of the groups of rational points of algebraic groups and their 1685 deformations

IGOR A. RAPINCHUK

Betti diagrams from graphs

AlEXANDER ENGSTRÖM and MATTHEW T. STAMPS

Hopf monoids from class functions on unitriangular matrices

Marcelo Aguiar, NANTEl Bergeron and Nathaniel Thiem 